\title{
Oxygen Binding of Water-Soluble Cobalt Porphyrins in Aqueous Solution
}

James P. Collman,* Yi-Long Yan, Todd Eberspacher, Xiangjin Xie, and

Edward I. Solomon

Department of Chemistry, Stanford University, California $94305-5080$

E-mail: ipc@,stanford.edu

\section{Supporting Information}

General Methods. Reagents and solvents were used as received unless otherwise indicated. Metallation reactions of porphyrin compounds were performed under $\mathrm{N}_{2}$ atmosphere. All air-sensitive samples for UV-vis and oxygenation measurements were prepared in a Vacuum Atmospheres glovebox (VAC) under inert atmosphere. Solvents and buffer solution for air-sensitive experiments were deoxygenated before use.

Preparation of compound 2:

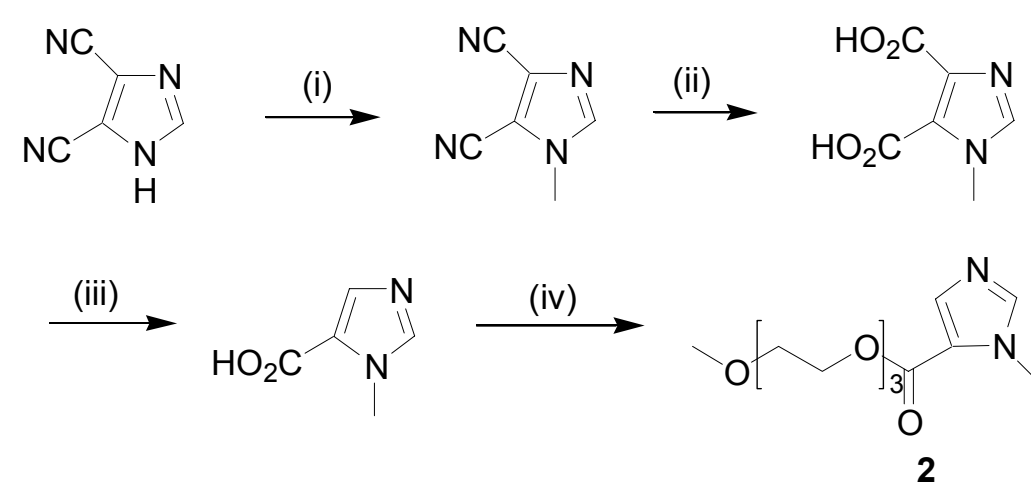

(i) $\left(\mathrm{CH}_{3} \mathrm{O}\right)_{2} \mathrm{SO}_{2}, \mathrm{NaHCO}_{3}$; (ii) (a) $2.0 \mathrm{~N} \mathrm{NaOH}$, (b) $\mathrm{HCl}$; (iii) $\mathrm{Ac}_{2} \mathrm{O}, 95{ }^{\circ} \mathrm{C}$; (iv) (a) $\mathrm{SOCl}_{2}, \mathrm{CH}_{3} \mathrm{CN}$, reflux, (b) triethylene glycol monomethyl ether, $\mathrm{CH}_{3} \mathrm{CN}$, reflux

1-methyl-5-imidazolecarboxylic acid was prepared following literature ${ }^{1}$. To a solution of 1-methyl-5-imidazolecarboxylic acid (400 mg, $3.17 \mathrm{mmol})$ in $\mathrm{CH}_{3} \mathrm{CN}$ (10 $\mathrm{mL}$ ) was added thionyl chloride $(3.0 \mathrm{~mL}, 30 \mathrm{mmol})$. The mixture was refluxed for $3 \mathrm{~h}$. Solvent and excess $\mathrm{SOCl}_{2}$ were evaporated under vacuum. The residue was redissolved in dry $\mathrm{CH}_{3} \mathrm{CN}(10 \mathrm{~mL})$. Triethylene glycol monomethyl ether $(0.7 \mathrm{~mL}, 4.14$ mmol) was added followed by triethylamine $(1.0 \mathrm{~mL}, 7.17 \mathrm{mmol})$. The mixture was refluxed for $4 \mathrm{~h}$. After concentration, the residue was purified by chromatography on 
silica gel with eluent $\mathrm{CH}_{3} \mathrm{OH} / \mathrm{CH}_{2} \mathrm{Cl}_{2}=5 / 95$ to give compound $2(540 \mathrm{mg}, 63 \%)$ as a light yellow oil. ${ }^{1} \mathrm{H}$ NMR $\left(500 \mathrm{MHz}, \mathrm{CDCl}_{3}\right): \delta 7.70(\mathrm{~s}, 1 \mathrm{H}), 7.59(\mathrm{~s}, 1 \mathrm{H}), 4.36(\mathrm{t}, J=$ $5.0 \mathrm{~Hz}, 2 \mathrm{H}), 3.86(\mathrm{~s}, 3 \mathrm{H}), 3.74(\mathrm{t}, J=4.5 \mathrm{~Hz}, 2 \mathrm{H}), 3.58-3.65(\mathrm{~m}, 6 \mathrm{H}), 3.47(\mathrm{t}, J=$ $4.5 \mathrm{~Hz}, 2 \mathrm{H}), 3.32$ (s, 3H); ${ }^{13} \mathrm{C} \mathrm{NMR}\left(\mathrm{CDCl}_{3}\right): 160.12,142.37,137.36,123.04,71.88$, $70.65,70.60,70.56,69.03,63.58,59.03,34.21$; MS (ESI): $\mathrm{m} / \mathrm{e}=272.8[\mathrm{M}+\mathrm{H}]^{+}$for $\mathrm{C}_{12} \mathrm{H}_{21} \mathrm{~N}_{2} \mathrm{O}_{5}$. HRMS (ESI) for $\mathrm{C}_{12} \mathrm{H}_{21} \mathrm{~N}_{2} \mathrm{O}_{5}[\mathrm{M}+\mathrm{H}]^{+}$: calcd. 273.1450, found 273.1461.

Preparation of compound 1Co: Porphyrin compound 1Co was prepared according following scheme.

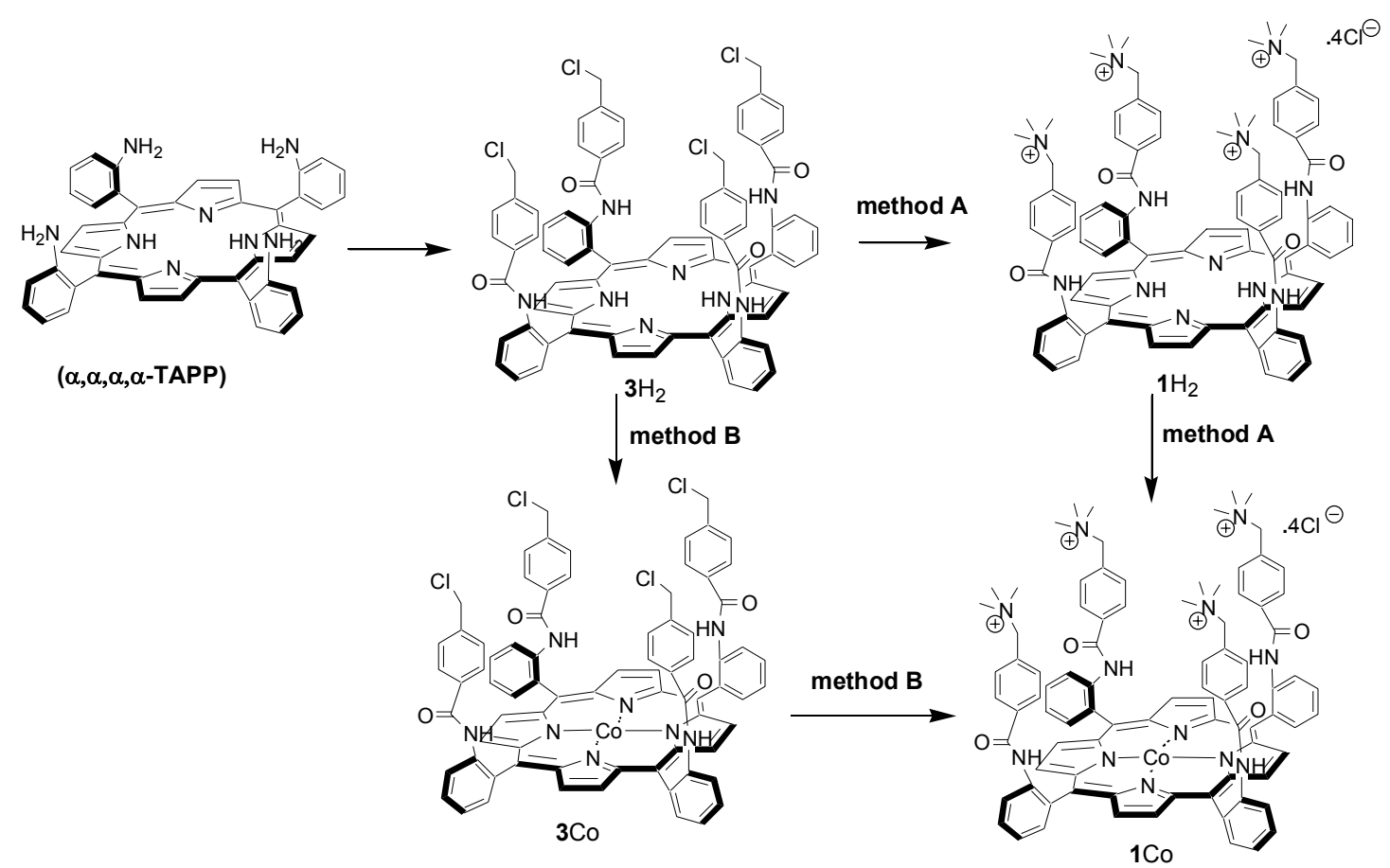

Preparation of compound $3 \mathbf{H}_{2}$ : $\alpha \alpha \alpha \alpha$-TAPP was prepared following the literature. ${ }^{2}$ $\alpha \alpha \alpha \alpha$-TAPP $(675 \mathrm{mg}, 1.0 \mathrm{mmol})$ was dissolved in dry $\mathrm{CH}_{2} \mathrm{Cl}_{2}(100 \mathrm{~mL})$. To this solution was added 4-Chloromethylbenzyl chloride $(1.51 \mathrm{~g}, 8.0 \mathrm{mmol})$ followed by $\mathrm{Et}_{3} \mathrm{~N}(1.12 \mathrm{~mL}, 8.0 \mathrm{mmol})$. The mixture was stirred at room temperature for $1 \mathrm{~h}$. The reaction mixture was washed with diluted $\mathrm{NaHCO}_{3}$ solution and dried over $\mathrm{MgSO}_{4}$. After concentration, the residue was purified by chromatography on silica gel with eluent EtOAc $/ \mathrm{CH}_{2} \mathrm{Cl}_{2}=8 / 92$ to give compound $3 \mathrm{H}_{2}(810 \mathrm{mg}, 63 \%)$ as a solid. ${ }^{1} \mathrm{H}$ NMR (500 MHz, $\left.\mathrm{CDCl}_{3}\right): \delta 8.96(\mathrm{~s}, 8 \mathrm{H}), 8.80(\mathrm{~d}, J=8.0 \mathrm{~Hz}, 4 \mathrm{H}), 8.00(\mathrm{~s}, 4 \mathrm{H}), 7.88$ $(\mathrm{t}, J=8.0 \mathrm{~Hz}, 4 \mathrm{H}), 7.80(\mathrm{~d}, J=7.5 \mathrm{~Hz}, 4 \mathrm{H}), 7.51(\mathrm{t}, J=7.5 \mathrm{~Hz}, 4 \mathrm{H}), 6.86(\mathrm{~d}, J=8.0$ $\mathrm{Hz}, 8 \mathrm{H}), 6.54(\mathrm{~d}, J=8 \mathrm{~Hz}, 8 \mathrm{H}), 3.92(\mathrm{~s}, 8 \mathrm{H}),-2.63(\mathrm{~s}, 2 \mathrm{H}) ;{ }^{13} \mathrm{C}$ NMR $\left(\mathrm{CDCl}_{3}\right)$ : $165.41,141.01,138.12,136.36,134.37,131.65,130.39,128.63,127.18,123.93$, 
122.15, 115.71, 45.05; MS (ESI): $\mathrm{m} / \mathrm{e}=1282.2\left(\mathrm{M}^{+}\right)$for $\mathrm{C}_{76} \mathrm{H}_{54} \mathrm{Cl}_{4} \mathrm{~N}_{8} \mathrm{O}_{4}$. HRMS (ESI) for $\mathrm{C}_{76} \mathrm{H}_{55} \mathrm{Cl}_{4} \mathrm{~N}_{8} \mathrm{O}_{4}[\mathrm{M}+\mathrm{H}]^{+}$: calcd. 1283.3100, found 1283.3102; UV-vis $\left(\mathrm{CH}_{2} \mathrm{Cl}_{2}\right)$ : $422,514 \mathrm{~nm}$.

Preparation of compound $\mathbf{1} \mathbf{H}_{\mathbf{2}}$ (Method A): Compound $\mathbf{3} \mathrm{H}_{2}(180 \mathrm{mg}, 0.14 \mathrm{mmol}$ ) was stirred in a methylamine solution in ethyl alcohol $(33 \%, 15 \mathrm{~mL})$ overnight at room temperature under $\mathrm{N}_{2}$ atmosphere. The mixture was concentrated under vacuum. The residue was recrystallized from $\mathrm{CH}_{3} \mathrm{OH} /$ Acetone solvent to give compound $\mathbf{1 H}_{2}$ as a solid (173 mg, 83\%). ${ }^{1} \mathrm{H}$ NMR (500 MHz, DMSO-d6): $\delta 10.10$ (s, 4H), 8.82 (s, $8 \mathrm{H}), 7.88(\mathrm{~d}, J=7.5 \mathrm{~Hz}, 4 \mathrm{H}), 7.84(\mathrm{t}, J=7.5 \mathrm{~Hz}, 4 \mathrm{H}), 7.73(\mathrm{~d}, J=7.5 \mathrm{~Hz}, 4 \mathrm{H}), 7.60$ (t, $J=7.5 \mathrm{~Hz}, 4 \mathrm{H}), 7.17(\mathrm{~d}, J=7.5 \mathrm{~Hz}, 8 \mathrm{H}), 7.10(\mathrm{~d}, J=7.5 \mathrm{~Hz}, 8 \mathrm{H}), 4.59$ (s, 8H), $2.89\left(\mathrm{~s}, 36 \mathrm{H}, \mathrm{NMe}_{3}\right),-2.99$ (s, 2H); ${ }^{13} \mathrm{C} \mathrm{NMR}\left(\mathrm{CD}_{3} \mathrm{OD}\right): 167.27,138.08,136.94$, $136.50,132.79,130.85,129.50,127.76,126.44,125.43,116.64,67.85,52.11$; MS (ESI): $\mathrm{m} / \mathrm{e}=1521.8\left(\mathrm{M}^{+}\right)$for $\mathrm{C}_{88} \mathrm{H}_{90} \mathrm{Cl}_{4} \mathrm{~N}_{12} \mathrm{O}_{4}$; HRMS (ESI) for $\mathrm{C}_{88} \mathrm{H}_{90} \mathrm{Cl}_{3} \mathrm{~N}_{12} \mathrm{O}_{4}$ $[\mathrm{M}-\mathrm{Cl}]^{+}$: calcd. 1483.6274, found 1483.6263; UV-vis $\left(\mathrm{CH}_{3} \mathrm{OH}\right): 422,520 \mathrm{~nm}$.

Preparation of compound 1Co: The reaction was carried out under a $\mathrm{N}_{2}$ atmosphere. To a solution of $1 \mathrm{H}_{2}(150 \mathrm{mg}, 0.1 \mathrm{mmol})$ in dry $\mathrm{CH}_{2} \mathrm{Cl}_{2} / \mathrm{CH}_{3} \mathrm{OH}(\mathrm{v} / \mathrm{v} 5: 1,30 \mathrm{~mL})$ was added $\mathrm{CoCl}_{2}$ (65 mg, $0.5 \mathrm{mmol}$ ). The mixture was gently refluxed overnight under $\mathrm{N}_{2}$. Completion of the reaction was checked by UV-vis spectra of an aliquot sample of the reaction mixture. After filtration, the filtrate was concentrated. The residue was dried under high vacuum to give product $1 \mathrm{Co}$ as a solid in quantitative yield. MS (ESI): $\mathrm{m} / \mathrm{e}$ $=1575[\mathrm{M}]^{+}$for $\mathrm{C}_{88} \mathrm{H}_{88} \mathrm{Cl}_{4} \mathrm{CoN}_{12} \mathrm{O}_{4}$; HRMS (ESI) for $\mathrm{C}_{88} \mathrm{H}_{88} \mathrm{Cl}_{3} \mathrm{CoN}_{12} \mathrm{O}_{4}[\mathrm{M}-\mathrm{Cl}]^{+}$: calcd. 1540.5449, found 1540.5419; UV-vis $\left(\mathrm{CH}_{3} \mathrm{OH}\right): 420,518 \mathrm{~nm}$. The product mixture was contaminated with a little unreacted $\mathrm{CoCl}_{2}$. As $1 \mathrm{Co}$ is very polar, the product could not be purified by chromatography on silica gel.

Preparation of compound $3 \mathrm{Co}(\operatorname{method} \mathrm{B})$ : The reaction was carried out under $\mathrm{N}_{2}$. To a solution of $3 \mathrm{H}_{2}(150 \mathrm{mg}, 0.12 \mathrm{mmol})$ in dry THF $(20 \mathrm{~mL})$ was added $\mathrm{CoCl}_{2}(150$ $\mathrm{mg}, 1.2 \mathrm{mmol})$ followed by 2,6 -lutidine $(350 \mu \mathrm{L}, 2.6 \mathrm{mmol})$. The mixture was stirred at room temperature for $8 \mathrm{~h}$. Completion of the reaction was checked by UV-vis spectra of an aliquot sample of the reaction mixture. After removal of solvent, the residue was dissolved in benzene $(60 \mathrm{~mL})$. The solution was washed with diluted 
ammonia solution, dried over $\mathrm{MgSO}_{4}$, and evaporated to dryness. The residue was purified by chromatography on silica gel with eluent EtOAc/ $\mathrm{CH}_{2} \mathrm{Cl}_{2}=8 / 92$ to give compound 3Co $(125 \mathrm{mg}, 82 \%)$ as a solid. $\mathrm{MS}(\mathrm{ESI}): \mathrm{m} / \mathrm{e}=1339.6[\mathrm{M}]^{+}$for $\mathrm{C}_{76} \mathrm{H}_{52} \mathrm{Cl}_{4} \mathrm{CoN}_{8} \mathrm{O}_{4}$; HRMS for $\mathrm{C}_{76} \mathrm{H}_{52} \mathrm{Cl}_{4} \mathrm{CoN}_{8} \mathrm{O}_{4}[\mathrm{M}]^{+}$calcd. 1339.2198, found 1339.2186; UV-Vis $\left(\mathrm{CH}_{2} \mathrm{Cl}_{2}\right)$ : 432, $548 \mathrm{~nm}$.

Preparation of compound 1Co from 3Co (method B): Compound 3Co (140 mg, $0.10 \mathrm{mmol})$ was stirred in a methylamine solution in ethyl alcohol $(33 \%, 15 \mathrm{~mL})$ overnight at room temperature under $\mathrm{N}_{2}$. Excess methylamine and solvent were evaporated off under vacuum. The residue was dried under high vacuum to give $1 \mathrm{Co}$ in quantitative yield.

\section{Reference:}

1. O’Connell, J. F.; Parquette, J.; Yelle, W. E.; Wang, W.; Rapoport, H. Synthesis, 1988, 767.

2. (a) Collman, J. P.; Gagne, R. R.; Reed, C.; Halbert, T. R.; Lang, G.; Robinson, W. T. J. Am. Chem. Soc. 1975, 97, 1427. (b) Lindsey, J. J. Org. Chem. 1980, $45,5215$. 


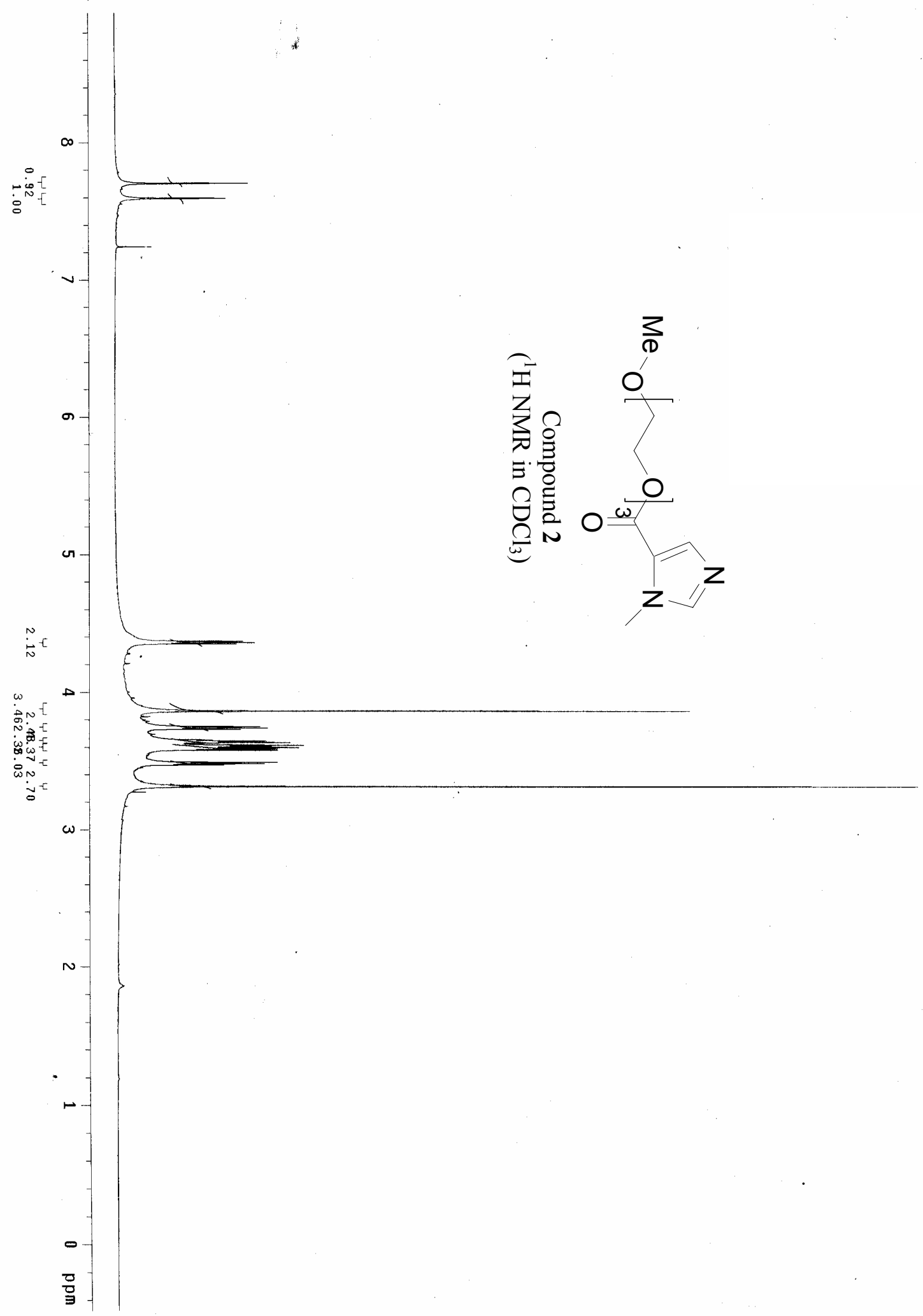




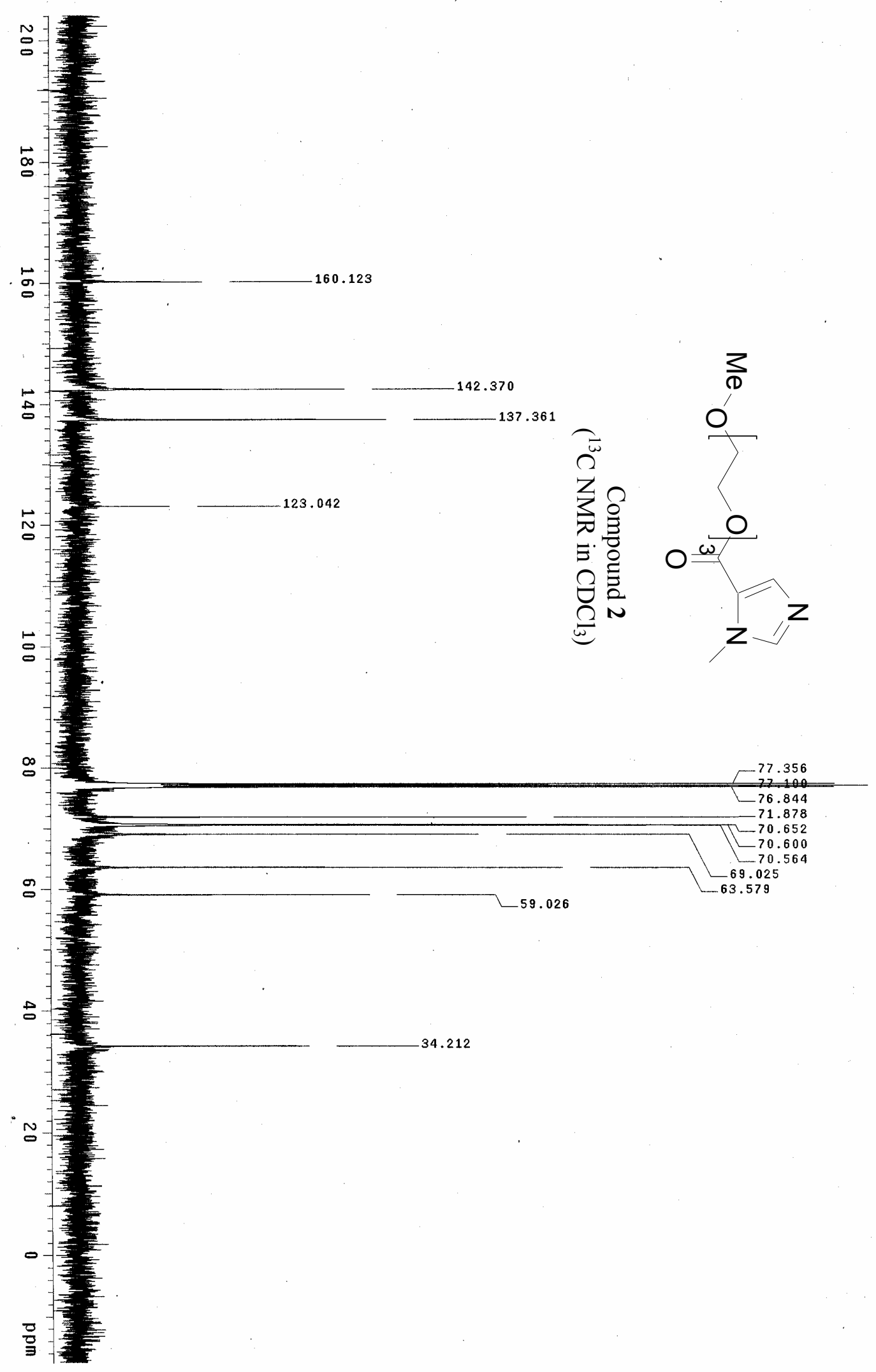




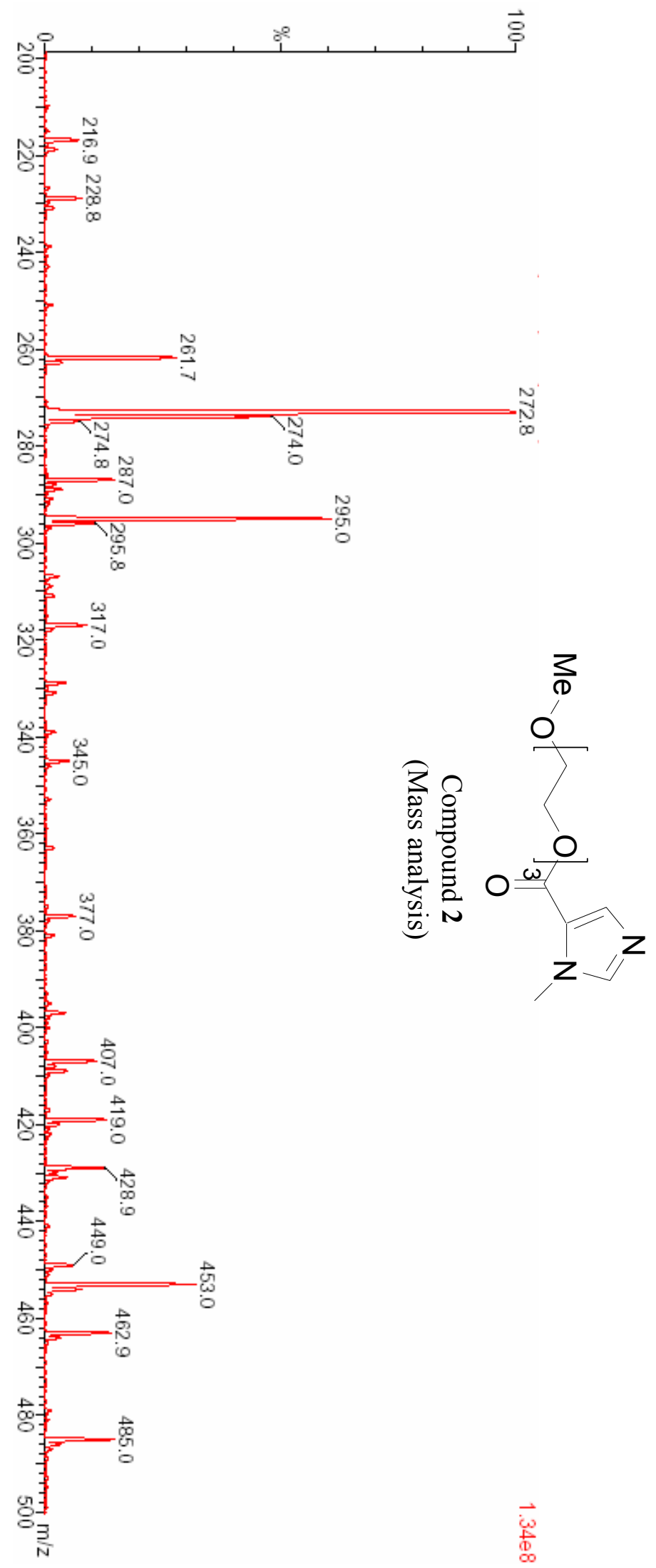




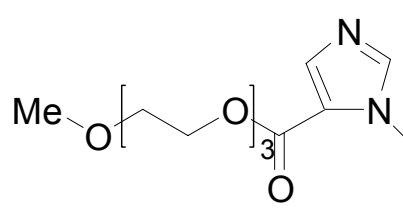

Compound 2 (High resolution mass analysis)

\section{Elemental Composition Report}

Single Mass Analysis

Tolerance $=5.0$ PPM / DBE: $\min =-1.5, \max =120.0$

Isotope cluster parameters: Separation $=1.0$ Abundance $=1.0 \%$

Monoisotopic Mass, Odd and Even Electron lons

408 formula(e) evaluated with 3 results within limits (up to 140 closest results for each mass)

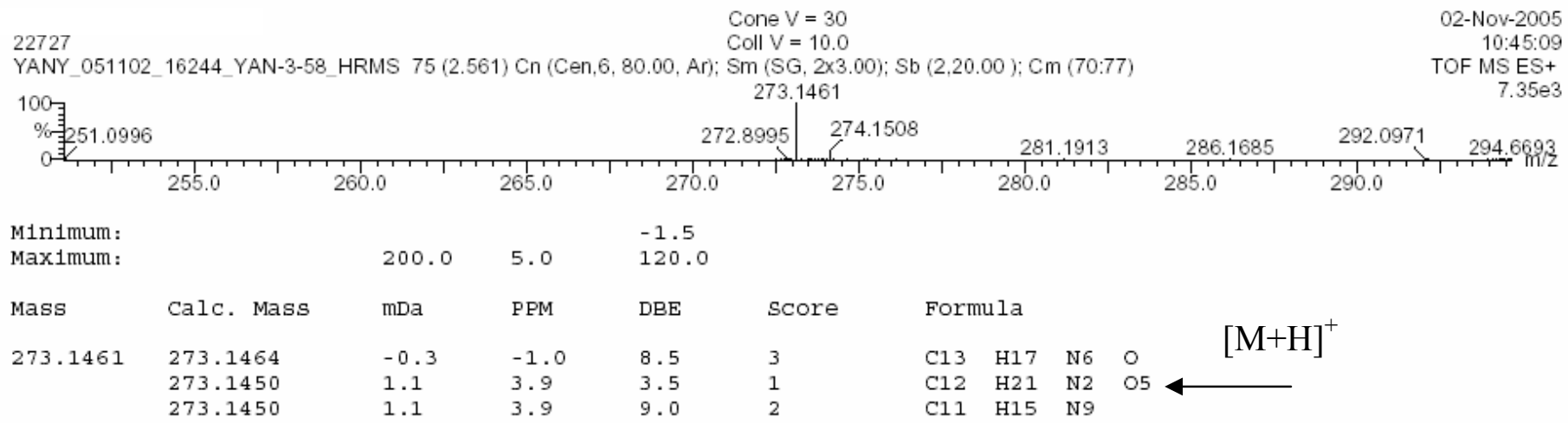




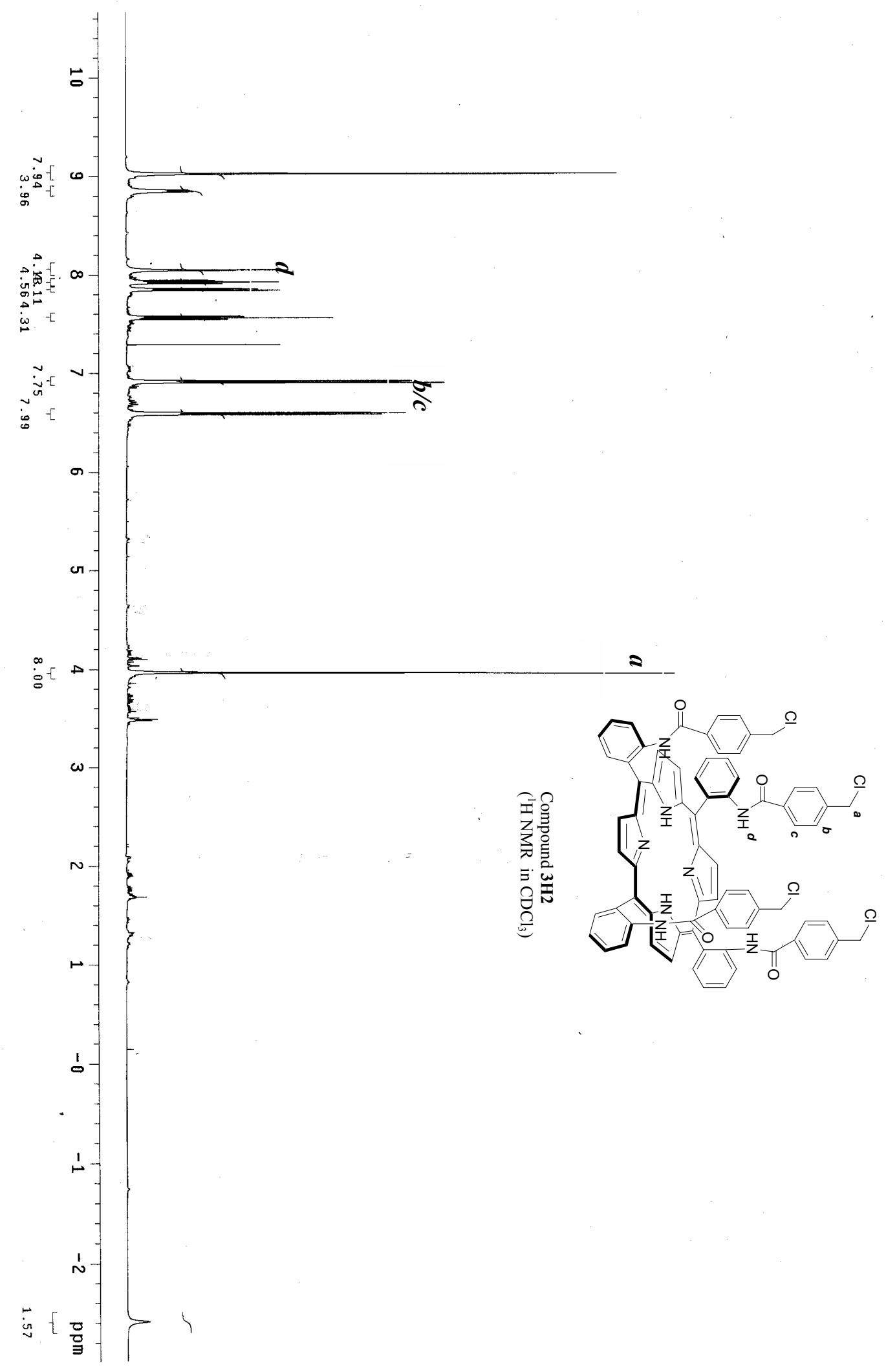




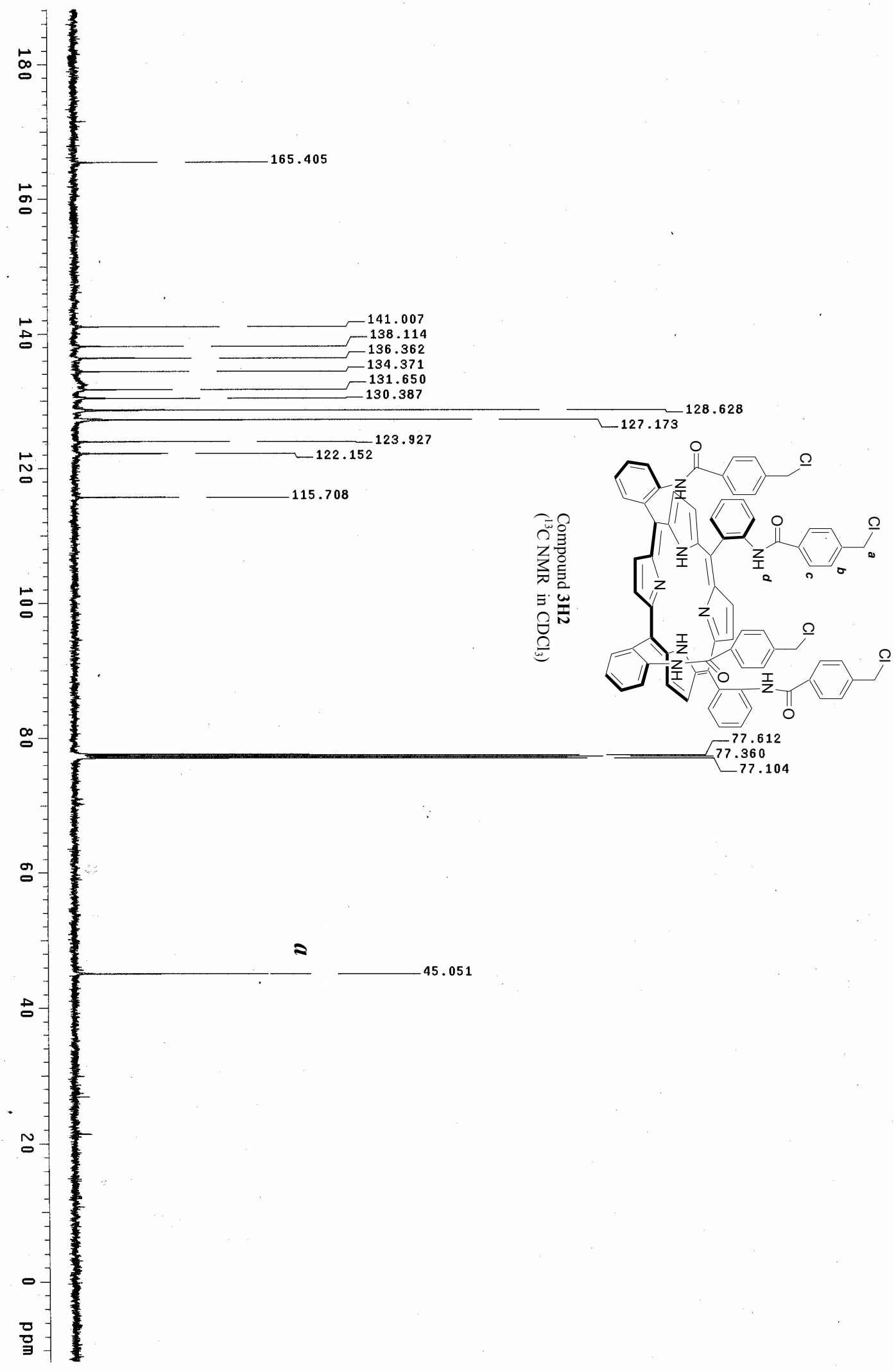




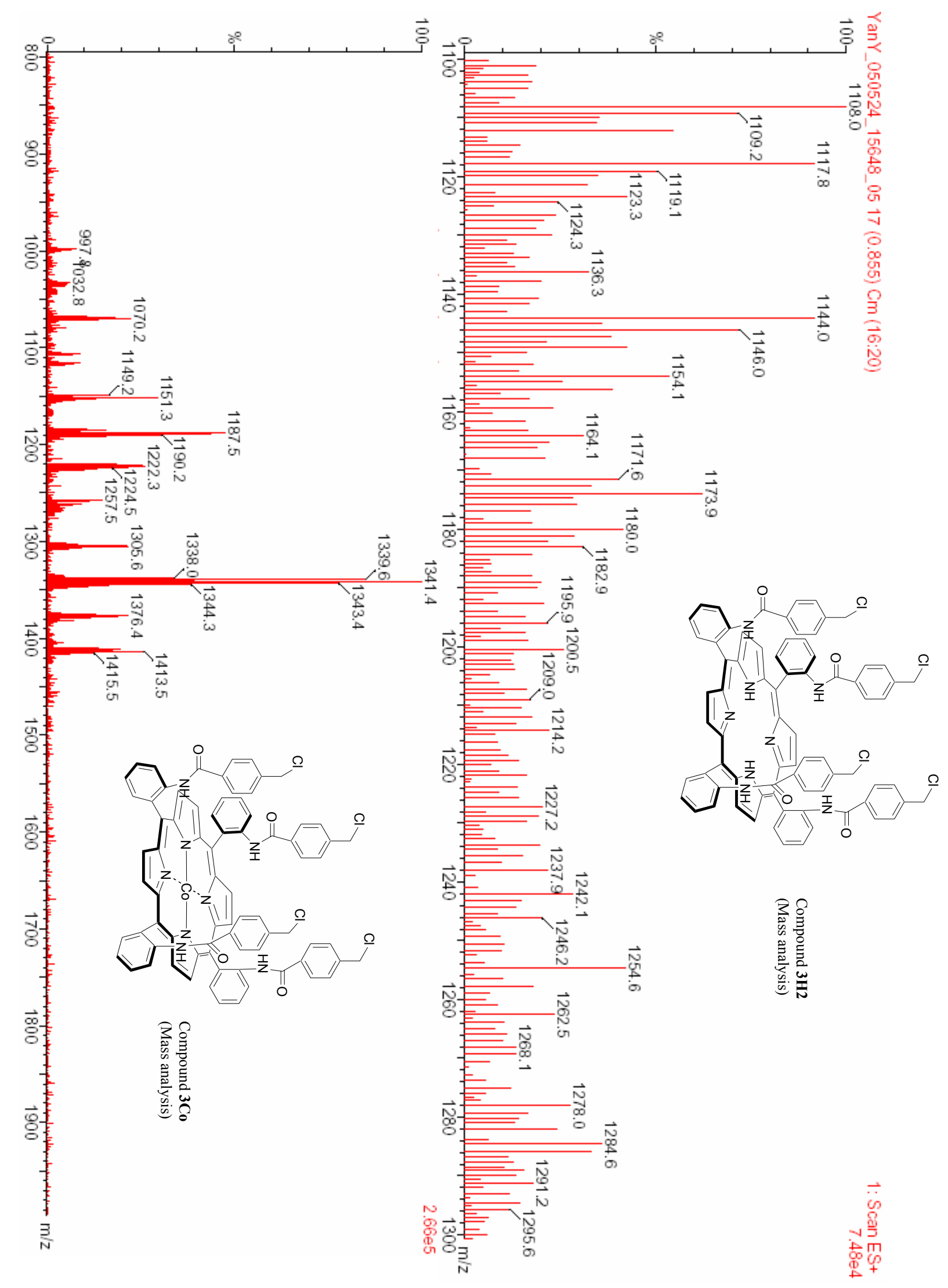




\section{Elemental Composition Report}

\section{Single Mass Analysis}

Tolerance $=5.0$ PPM / DBE: $\min =-1.5, \max =120.0$

Isotope cluster parameters: Separation $=1.0 \quad$ Abundance $=1.0 \%$

Monoisotopic Mass, Odd and Even Electron lons

17958 formula(e) evaluated with 237 results within limits (up to 140 closest results for each mass)

Yilong Yan, Yan-4-13

16129

Cone $V=25$

Coll $\mathrm{V}=10.0$

YANY 05110216244 YAN-4-13 HRMS 34 (1.166) Cn (Cen,6, 80.00, Ar): Sm (SG, 2x3.00); Sb (2.20.00): Cm (31:43)

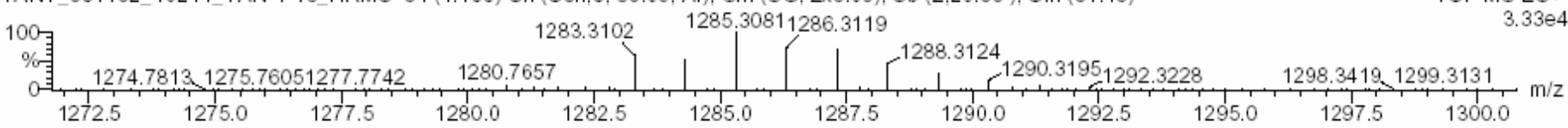

Minimum :

Maximum

Mass

Calc. Mass

1283.3102

1283.3103

1283.3100

1283.3100

1283.3104

1283.3104

1283.3104

1283.3099

1283.3099

1283.3099

1283.3099

1283.3099

1283.3105

1283.3098

283.3098

1283.3106

1283.3107

1283.3108

1283.3108

1283.3108

1283.3108

1283.3108

1283.3096

1283.3096

1283.3095

1283.3109

1283.3109

1283.3109

1283.3094

1283.3094

1283.3094

283.3094

1283.3094

1283.3094

1283.3093

1283.3093

1283.3111

1283.3111

1283.3112

1283.3113

1283.3113

1283.3113

1283.3113

1283.3091

1283.3091

1283.3114

1283.3114

1283.3090

1283.3089

1283.3089

1283.3088

1283.3116

1283.3116

1283.3116

1283.3087

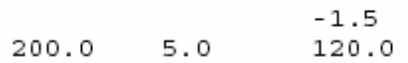

mDa PPM Score Formula

$\begin{array}{llll}-0.1 & -0.1 & 53.5 & 71\end{array}$

$\begin{array}{llll}-0.1 & -0.1 & 48.0 & 69\end{array}$

$\begin{array}{llll}0.2 & 0.1 & 46.0 & 2\end{array}$

$\begin{array}{llll}0.2 & 0.1 & 51.5 & 4\end{array}$

$\begin{array}{lll}-0.2 & -0.2 & 65.5\end{array}$

$-0.2-0.2 \quad 60.0$

$\begin{array}{lll}-0.2 & -0.2 & 57.5\end{array}$

$\begin{array}{lll}.3 & 0.2 & 62.0\end{array}$

$\begin{array}{lll}0.3 & 0.2 & 64.5\end{array}$

$\begin{array}{lll}0.3 & 0.2 & 39.5\end{array}$

$\begin{array}{lll}0.3 & 0.2 & 70.0\end{array}$

$\begin{array}{lll}0.3 & 0.2 & 45.0\end{array}$

$\begin{array}{lll}-0.3 & -0.3 & 39.0\end{array}$

$\begin{array}{lll}0.4 & 0.3 & 58.0\end{array}$

$\begin{array}{lll}0.4 & 0.3 & 63.5\end{array}$

$\begin{array}{lll}-0.4 & -0.3 & 67.5\end{array}$

$-0.5 \quad-0.4 \quad 62.0$

$\begin{array}{lll}-0.6 & -0.4 & 79.5\end{array}$

$\begin{array}{lll}-0.6 & -0.4 & 49.0\end{array}$

$\begin{array}{lll}-0.6 & -0.4 & 74.0\end{array}$

$\begin{array}{lll}-0.6 & -0.4 & 43.5\end{array}$

$\begin{array}{lll}-0.6 & -0.5 & 41.0\end{array}$

$\begin{array}{lll}0.6 & 0.5 & 50.5\end{array}$

$\begin{array}{lll}0.6 & 0.5 & 56.0\end{array}$

$\begin{array}{lll}0.7 & 0.5 & 61.5\end{array}$

$\begin{array}{lll}-0.7 & -0.5 & 61.0\end{array}$

$\begin{array}{lll}-0.7 & -0.5 & 55.5\end{array}$

$\begin{array}{lll}-0.7 & -0.6 & 53.0\end{array}$

$\begin{array}{lll}0.8 & 0.6 & 41.5\end{array}$

$\begin{array}{lll}0.8 & 0.6 & 69.0\end{array}$

$\begin{array}{lll}0.8 & 0.6 & 44.0\end{array}$

$\begin{array}{lll}0.8 & 0.6 & 74.5\end{array}$

$\begin{array}{lll}0.8 & 0.6 & 49.5\end{array}$

$\begin{array}{lll}0.8 & 0.6 & 52.0\end{array}$

$0.9 \quad 0.7 \quad 62.5$

$\begin{array}{lll}0.9 & 0.7 & 68.0\end{array}$

$\begin{array}{lll}-0.9 & -0.7 & 63.0\end{array}$

$\begin{array}{lll}-0.9 & -0.7 & 57.5\end{array}$

$\begin{array}{lll}-1.0 & -0.7 & 55.0\end{array}$

$\begin{array}{lll}-1.1 & -0.8 & 44.5\end{array}$

$-1.1$

$-1.1$

$-1.1$

1.1

1.1

$-1.2$

$-1.2$

1.2

$1 \cdot 3$
$1 \cdot 4$

$-1.4$

$-1.4$

$-1.4$

$-0.8$

$-0.8$

$-0.8$

0.9

0.9

$-0.9$

$-0.9$

1. 0

1.0

1.0

1.1

$-1.1$

$-1.1$

$-1.1$

69.5

39.0

36.5

60.5

66.0

56.5

51.0

46.0

48.5

54.0

75.0

8.5

53.0

50.5

44.0
02-Nov-2005 11:06:38 MS ES+

$33 e 4$

$\begin{array}{llll}1292.5 & 1295.0 & 1297.5 & 1300.0\end{array}$

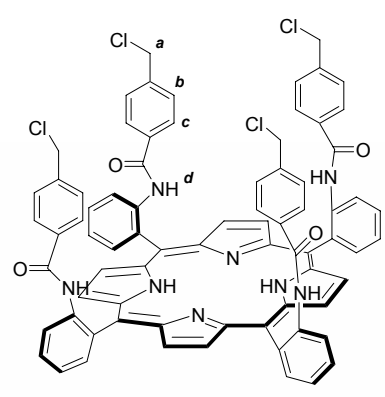

N

Page 1

High resoluti

Mass analysis) 


\section{Elemental Composition Report}

\section{Single Mass Analysis}

Tolerance $=5.0$ PPM / DBE: $\min =-1.5, \max =120.0$ Isotope cluster parameters: Separation $=1.0$ Abundance $=1.0 \%$

Monoisotopic Mass, Odd and Even Electron lons 17958 formula(e) evaluated with 237 results within limits (up to 140 closest results for each mass)

Yilong Yan, Yan-4-13

16129

YANY_051102_16244_YAN-4-13_HRMS 34 (1.166) Cn (Cen,6, 80.00, Ar); Sm (SG, 2×3.00); Sb $(2,20.00) ; \mathrm{Cm}(31: 43)$

$\begin{array}{rrr}100 & 1283.3102 & 1285.30811286 .3119\end{array}$

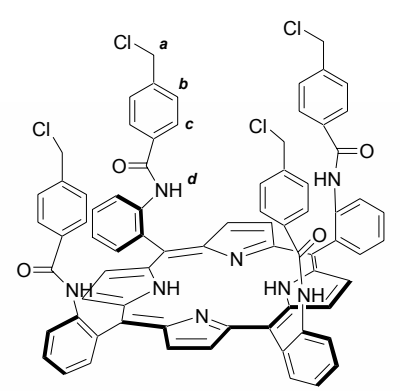

$=0$

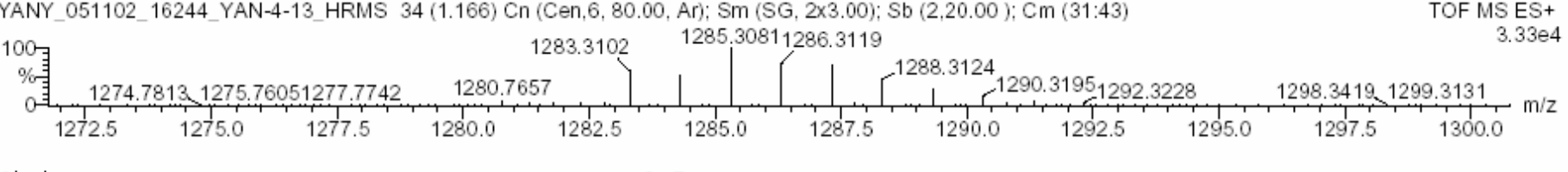

Minimum :

Maximum :

Mass

Calc. Mass

$200.0 \quad 5.0$

$-1.5$

1283.3087

1283.3087

1283.3117

1283.3117

1283.3118

1283.3118

1283.3086

1283.3086

1283.3086

1283.3086

1283.3086

1283.3086

1283.3085

1283.3085

1283.3120

1283.3121

1283.3121

1283.3121

1283.3082

1283.3082

1283.3122

1283.3122

1283.3123

1283.3081

1283.3081

1283.3081

1283.3081

1283.3081

1283.3080

1283.3080

1283.3080

1283.3125

1283.3125

1283.3125

1283.3126

1283.3126

1283.3126

1283.3126

1283.3126

1283.3077

1283.3077

1283.3127

1283.3127

1283.3127

1283.3076

1283.3076

1283.3076

1283.3130

1283.3130

1283.3130

1283.3074

1283.3074

1283.3131

1283.3131

1283.3131

1283.3072

\begin{tabular}{|c|c|c|c|}
\hline $\mathrm{mDa}$ & P PM & DBE & score \\
\hline 1.5 & 1.2 & 46.5 & 5 \\
\hline 1.5 & 1.2 & 52.0 & 8 \\
\hline-1.5 & -1.2 & 65.0 & 59 \\
\hline-1.5 & -1.2 & 59.5 & 58 \\
\hline-1.6 & -1.2 & 32.0 & 26 \\
\hline-1.6 & -1.2 & 57.0 & 84 \\
\hline 1.6 & 1.3 & 34.5 & 18 \\
\hline 1.6 & 1.3 & 65.0 & 98 \\
\hline 1.6 & 1.3 & 40.0 & 19 \\
\hline 1.6 & 1.3 & 70.5 & 107 \\
\hline 1.6 & 1.3 & 45.5 & 20 \\
\hline 1.6 & 1.3 & 73.0 & 55 \\
\hline 1.7 & 1.4 & 53.0 & 85 \\
\hline 1.7 & 1.4 & 58.5 & 93 \\
\hline-1.8 & -1.4 & 67.0 & 125 \\
\hline-1.9 & -1.5 & 79.0 & 117 \\
\hline-1.9 & -1.5 & 48.5 & 42 \\
\hline-1.9 & -1.5 & 73.5 & 112 \\
\hline 2.0 & 1.5 & 51.0 & 35 \\
\hline 2.0 & 1.6 & 56.5 & 37 \\
\hline-2.0 & -1.6 & 60.5 & 30 \\
\hline-2.0 & -1.6 & 55.0 & 28 \\
\hline-2.1 & -1.6 & 52.5 & 77 \\
\hline 2.1 & 1.6 & 69.5 & 118 \\
\hline 2.1 & 1.6 & 44.5 & 47 \\
\hline 2.1 & 1.6 & 75.0 & 123 \\
\hline 2.1 & 1.6 & 50.0 & 48 \\
\hline 2.1 & 1.7 & 77.5 & 82 \\
\hline 2.2 & 1.7 & 57.5 & 127 \\
\hline 2.2 & 1.7 & 63.0 & 131 \\
\hline 2.2 & 1.7 & 68.5 & 135 \\
\hline-2.3 & -1.8 & 62.5 & 103 \\
\hline-2.3 & -1.8 & 57.0 & 94 \\
\hline$-2 \cdot 3$ & -1.8 & 54.5 & 139 \\
\hline-2.4 & -1.9 & 74.5 & 110 \\
\hline-2.4 & -1.9 & 44.0 & 12 \\
\hline-2.4 & -1.9 & 69.0 & 111 \\
\hline-2.4 & -1.9 & 66.5 & 133 \\
\hline-2.4 & -1.9 & 36.0 & 53 \\
\hline 2.5 & 1.9 & 55.5 & 63 \\
\hline 2.5 & 1.9 & 61.0 & 65 \\
\hline-2.5 & -2.0 & 56.0 & 6 \\
\hline-2.5 & -2.0 & 50.5 & 1 \\
\hline-2.5 & -2.0 & 48.0 & 50 \\
\hline 2.6 & 2.0 & 49.0 & 75 \\
\hline 2.6 & 2.0 & 54.5 & 76 \\
\hline 2.6 & 2.0 & 57.0 & 29 \\
\hline-2.8 & -2.2 & 58.0 & 67 \\
\hline-2.8 & -2.2 & 52.5 & 66 \\
\hline-2.8 & -2.2 & 50.0 & 90 \\
\hline 2.8 & 2.2 & 47.0 & 9 \\
\hline 2.8 & 2.2 & 52.5 & 11 \\
\hline-2.9 & -2.2 & 64.5 & 56 \\
\hline-2.9 & $-2 \cdot 3$ & 62.0 & 89 \\
\hline-2.9 & $-2 \cdot 3$ & 31.5 & 25 \\
\hline 3.0 & 2.3 & 35.0 & 21 \\
\hline
\end{tabular}

Formula

$\begin{array}{lllll}\mathrm{C} 75 & \mathrm{H} 59 & \mathrm{~N} 4 & 08 & \mathrm{Cl} 4\end{array}$

$\mathrm{C} 74 \mathrm{H} 53$ N11 $\mathrm{O}_{3} \mathrm{Cl}$

$\begin{array}{llll}\mathrm{C} 86 & \mathrm{H} 53 & \mathrm{O} 8 & \mathrm{Cl} 2\end{array}$

$\begin{array}{rrlrrr}\mathrm{C} 86 & \mathrm{H} 53 & 08 & \mathrm{Cl} 2 & & \\ \mathrm{C} 58 & \mathrm{H} 64 & \mathrm{~N} 14 & 08 & \mathrm{Cl} 4 & \mathrm{CO}\end{array}$

C74 H46 N11 $010 \quad \mathrm{Cl}$

$\begin{array}{llllll}\mathrm{C} 70 & \mathrm{H} 70 & \mathrm{~N} & 010 & \mathrm{Cl} 4 & \mathrm{CO}\end{array}$

$\begin{array}{lllll}\mathrm{C} 85 & \mathrm{H} 46 & \mathrm{~N} 5 & 07 & \mathrm{Cl}\end{array}$

$\begin{array}{llllll}\mathrm{C} 69 & \mathrm{H} 64 & \mathrm{~N} 8 & \mathrm{O} & \mathrm{Cl} 4 & \mathrm{CO}\end{array}$

$\mathrm{C} 84 \mathrm{H} 40 \quad \mathrm{~N} 12$ O2 $\mathrm{Cl}$

$\begin{array}{lllll}\mathrm{C} 68 & \mathrm{H} 58 & \mathrm{~N} 15 & \mathrm{Cl} 4 & \mathrm{CO}\end{array}$

$\begin{array}{llll}\mathrm{C} 96 & \mathrm{H} 47 & \mathrm{~N} & \mathrm{Cl} 2\end{array}$

$\begin{array}{llllll}\mathrm{C} 80 & \mathrm{H} 57 & \mathrm{~N} 2 & \mathrm{O} 9 & \mathrm{Cl} & \mathrm{CO}\end{array}$

C79 H51 N9 O4 Cl Co

$\begin{array}{lllll}\mathrm{C} 87 & \mathrm{H} 48 & \mathrm{~N} 6 & \mathrm{O} 3 & \mathrm{CO}\end{array}$

C92 H37 N9 O

$\begin{array}{llllll}\mathrm{C} 77 & \mathrm{H} 61 & \mathrm{~N} 5 & \mathrm{O} 4 & \mathrm{Cl} 3 & \mathrm{CO}\end{array}$

C93 H43 N2 O6

$\begin{array}{lllll}\mathrm{C} 78 & \mathrm{H} 56 & \mathrm{~N} 3 & \mathrm{O} & \mathrm{Cl}\end{array}$

$\begin{array}{lllll}\mathrm{C} 77 & \mathrm{H} 50 & \mathrm{~N} 10 & \mathrm{O} 4 & \mathrm{Cl} 3\end{array}$

$\begin{array}{lllll}\mathrm{C} 82 & \mathrm{H} 50 & \mathrm{~N} 8 & \mathrm{O} 2 & \mathrm{Cl} 3\end{array}$

$\begin{array}{lllll}\mathrm{C} 83 & \mathrm{H} 56 & \mathrm{~N} & \mathrm{O} & \mathrm{Cl} 3\end{array}$

$\begin{array}{lllll}\mathrm{C} 71 & \mathrm{H} 49 & \mathrm{~N} 12 & \mathrm{O} 9 & \mathrm{Cl} 2\end{array}$

$\begin{array}{llll}\mathrm{C} 88 & \mathrm{H} 43 & \mathrm{~N} 4 & \mathrm{O}\end{array}$

$\begin{array}{llllll}\mathrm{C} 72 & \mathrm{H} 61 & \mathrm{~N} 7 & \mathrm{C} & \mathrm{Cl} & \mathrm{CO}\end{array}$

$\begin{array}{llll}\mathrm{C} 87 & \mathrm{H} 37 & \mathrm{~N} 11 & \mathrm{O} 3\end{array}$

C71 H55 N14 O Cl3 Co

$\mathrm{C} 99 \mathrm{H} 44$ O $\mathrm{Cl}$

$\mathrm{C} 83 \mathrm{H} 54$ N 010 CO

C82 H48 N8 O5 Co

C81 H42 N15 CO

$\mathrm{C} 84$ H51 $\mathrm{N} 7$ O2 $\mathrm{Cl}$ CO

$\begin{array}{lllll}\mathrm{C} 85 & \mathrm{H} 57 & \mathrm{O} 7 & \mathrm{Cl} & \mathrm{Co}\end{array}$

C73 H50 N11 O9 CO

C89 H4O N10 Cl

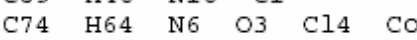

$\mathrm{C} 90 \mathrm{H} 46$ N3 05 Cl

$\begin{array}{llll}\mathrm{C} 78 & \mathrm{H} 39 & \mathrm{~N} 14 & 07\end{array}$

$\begin{array}{llllll}\mathrm{C} 63 & \mathrm{H} 63 & \mathrm{~N} 10 & 010 & \mathrm{Cl} 3 & \mathrm{CO}\end{array}$

$\begin{array}{lllll}\mathrm{C} 81 & \mathrm{H} 53 & \mathrm{~N} 2 & \mathrm{O} 10 & \mathrm{Cl} 2\end{array}$

$\begin{array}{lllll}\mathrm{C} 80 & \mathrm{H} 47 & \mathrm{~N} 9 & \mathrm{O} & \mathrm{Cl} 2\end{array}$

C79 H53 N9 O $\mathrm{Cl} 4$

$\begin{array}{lllll}\mathrm{C} 80 & \mathrm{H} 59 & \mathrm{~N} 2 & \mathrm{O} & \mathrm{Cl}\end{array}$

$\begin{array}{lllll}\mathrm{C} 68 & \mathrm{H} 52 & \mathrm{~N} 13 & 08 & \mathrm{Cl} 3\end{array}$

$\begin{array}{llllll}\mathrm{C} 75 & \mathrm{H} 58 & \mathrm{~N} 6 & \mathrm{O} & \mathrm{Cl} 2 & \mathrm{CO}\end{array}$

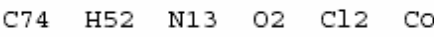

C86 H59 N2 $\mathrm{Cl} 3 \mathrm{CO}$

$\begin{array}{llllll}\mathrm{C} 81 & \mathrm{H} 54 & \mathrm{~N} 8 & \mathrm{O} & \mathrm{Cl} 2 & \mathrm{CO}\end{array}$

$\begin{array}{llllll}\mathrm{C} 82 & \mathrm{H} 60 & \mathrm{~N} & \mathrm{O} & \mathrm{Cl} 2 & \mathrm{CO}\end{array}$

$\begin{array}{llllll}\mathrm{C} 70 & \mathrm{H} 53 & \mathrm{~N} 12 & 08 & \mathrm{Cl} & \mathrm{CO}\end{array}$

$\begin{array}{lllll}\mathrm{C} 73 & \mathrm{H} 57 & \mathrm{~N} 7 & \mathrm{O} 7 & \mathrm{Cl} 4\end{array}$

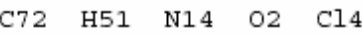

$\begin{array}{lllll}\mathrm{C} 87 & \mathrm{H} 49 & \mathrm{~N} 4 & \mathrm{O} 4 & \mathrm{Cl} 2\end{array}$

$\begin{array}{lllll}\mathrm{C} 75 & \mathrm{H} 42 & \mathrm{~N} 15 & \mathrm{O} & \mathrm{Cl}\end{array}$

$\begin{array}{llllll}\mathrm{C} 60 & \mathrm{H} 66 & \mathrm{~N} 11 & 09 & \mathrm{Cl} 4 & \mathrm{CO}\end{array}$

C68 $\mathrm{H} 68$ N4 09 Cl4 Co
02-Nov-2005 11:06:38 3. $33 \mathrm{e} 4$ 


\section{Elemental Composition Report}

\section{Single Mass Analysis}

Tolerance $=5.0$ PPM / DBE: $\min =-1.5, \max =120.0$

Isotope cluster parameters: Separation $=1.0$ Abundance $=1.0 \%$

Monoisotopic Mass, Odd and Even Electron Ions

17958 formula(e) evaluated with 237 results within limits (up to 140 closest results for each mass)

Yilong Yan, Yan-4-13

16129

Cone $\mathrm{V}=25$

Coll $V=10.0$

YANY_051102_16244_YAN-4-13_HRMS $34(1.166) \mathrm{Cn}(\mathrm{Cen}, 6,80.00, \mathrm{Ar}) ; \mathrm{Sm}(\mathrm{SG}, 2 \times 3.00) ; \mathrm{Sb}(2,20.00) ; \mathrm{Cm}(31: 43)$

100 年 $\quad 1283.3102 \quad 1285.30811286 .3119$

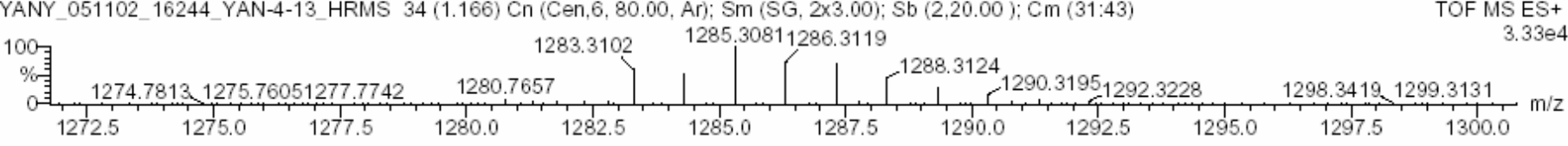

Minimum :

Maximum :

Mass

$\begin{array}{lll}200.0 & 5.0 & -1.5 \\ & & 120.0\end{array}$

Calc. Mass

Formula

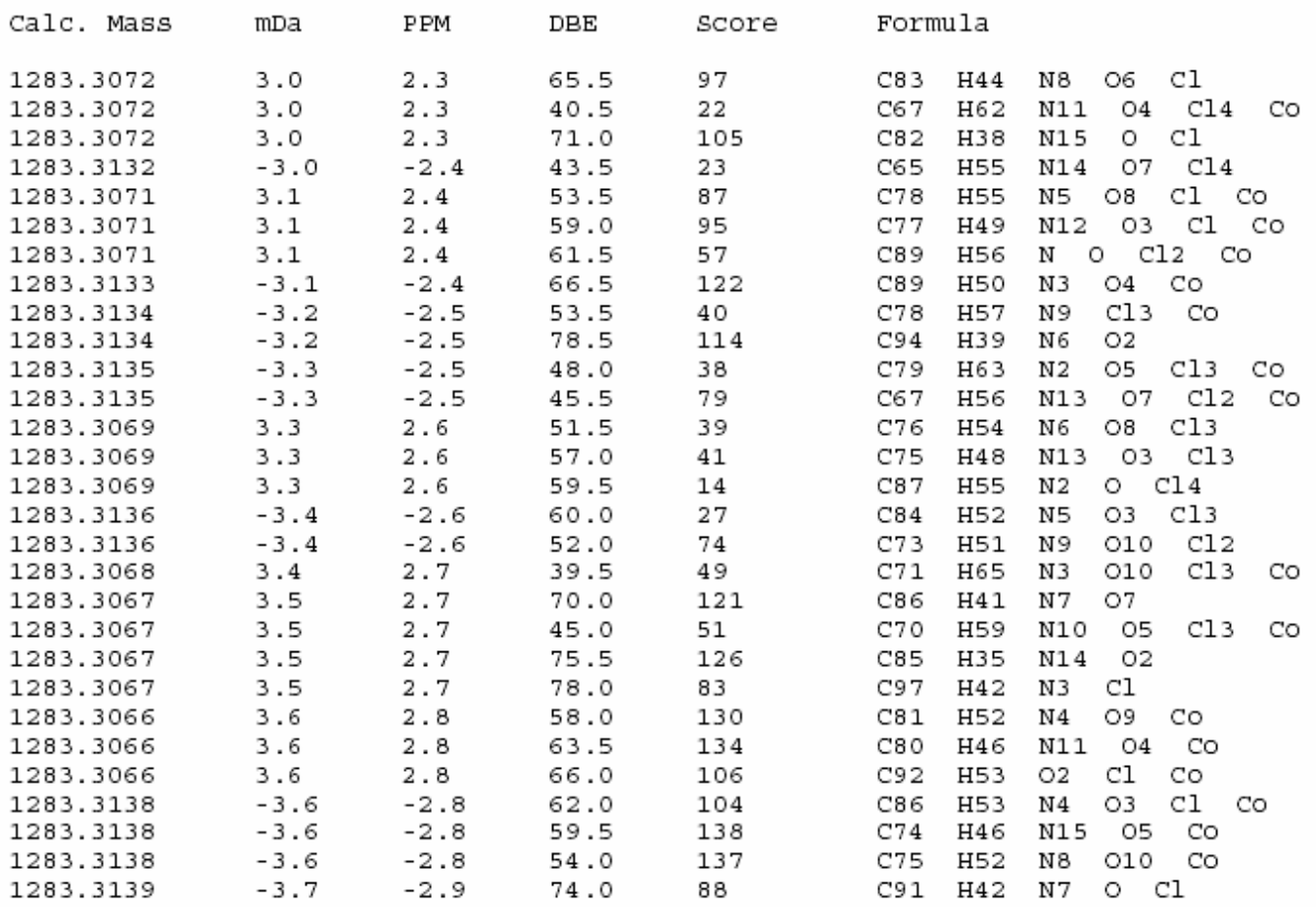

\begin{tabular}{|c|c|c|c|c|c|c|c|c|c|c|}
\hline 1283.3072 & 3.0 & 2.3 & 65.5 & 97 & $\mathrm{C} 83$ & $\mathrm{H} 44$ & N8 & 06 & \multirow{2}{*}{$\begin{array}{l}\mathrm{Cl} \\
\mathrm{Cl} 4\end{array}$} & \\
\hline 1283.3072 & 3.0 & 2.3 & 40.5 & 22 & $\mathrm{C} 67$ & $\mathrm{H} 62$ & N11 & 04 & & $\mathrm{CO}$ \\
\hline 1283.3072 & 3.0 & 2.3 & 71.0 & 105 & $\mathrm{C} 82$ & H38 & N15 & 0 & Cl4 & \\
\hline 1283.3132 & -3.0 & -2.4 & 43.5 & 23 & C65 & H55 & N14 & 07 & $\mathrm{Cl} 4$ & \\
\hline 1283.3071 & 3.1 & 2.4 & 53.5 & 87 & $\mathrm{C} 78$ & H55 & N5 & 08 & $\mathrm{Cl}$ & $\mathrm{CO}$ \\
\hline 1283.3071 & 3.1 & 2.4 & 59.0 & 95 & $\mathrm{C} 77$ & $\mathrm{H} 49$ & N12 & 03 & \multicolumn{2}{|c|}{$\mathrm{Cl} \mathrm{Co}$} \\
\hline 1283.3071 & 3.1 & 2.4 & 61.5 & 57 & $\mathrm{C} 89$ & H5 6 & $\mathrm{~N}$ & O C & $12 \mathrm{c}$ & Co \\
\hline 1283.3133 & -3.1 & -2.4 & 66.5 & 122 & $\mathrm{C} 89$ & $\mathrm{H} 50$ & N3 & $\bigcirc 4$ & \multicolumn{2}{|l|}{ Co } \\
\hline 1283.3134 & -3.2 & -2.5 & 53.5 & 40 & $\mathrm{C} 78$ & H5 7 & N9 & $\mathrm{Cl3}$ & $\mathrm{CO}$ & \\
\hline 1283.3134 & -3.2 & -2.5 & 78.5 & 114 & $\mathrm{C} 94$ & H39 & N6 & $\mathrm{O} 2$ & \multirow[b]{2}{*}{$\mathrm{Cl} 3$} & \\
\hline 1283.3135 & $-3 \cdot 3$ & -2.5 & 48.0 & 38 & C79 & $\mathrm{H} 63$ & N2 & 05 & & Co \\
\hline 1283.3135 & $-3 \cdot 3$ & -2.5 & 45.5 & 79 & $\mathrm{C} 67$ & $\mathrm{H} 56$ & N13 & 07 & $\mathrm{Cl} 2$ & $2 \mathrm{CO}$ \\
\hline 1283.3069 & 3.3 & 2.6 & 51.5 & 39 & $\mathrm{C} 76$ & H5 4 & N6 & 08 & $\mathrm{Cl3}$ & \\
\hline 1283.3069 & 3.3 & 2.6 & 57.0 & 41 & C75 & $\mathrm{H} 48$ & N13 & 03 & $\mathrm{Cl} 3$ & \\
\hline 1283.3069 & 3.3 & 2.6 & 59.5 & 14 & $\mathrm{C} 87$ & H55 & $\mathrm{N} 2$ & 0 & $\mathrm{Cl} 4$ & \\
\hline 1283.3136 & -3.4 & -2.6 & 60.0 & 27 & $\mathrm{C} 84$ & $\mathrm{H} 52$ & N5 & 03 & Cl3 & \\
\hline 1283.3136 & -3.4 & -2.6 & 52.0 & 74 & $\mathrm{C} 73$ & H51 & N9 & 010 & $\mathrm{Cl} 2$ & \\
\hline 1283.3068 & 3.4 & 2.7 & 39.5 & 49 & C71 & $\mathrm{H} 65$ & N3 & 010 & $\mathrm{Cl} 3$ & $\mathrm{CO}$ \\
\hline 1283.3067 & 3.5 & 2.7 & 70.0 & 121 & C86 & H4 1 & N7 & 07 & \multirow{3}{*}{$\mathrm{Cl} 3$} & \\
\hline 1283.3067 & 3.5 & 2.7 & 45.0 & 51 & $\mathrm{C} 7 \mathrm{O}$ & H59 & N10 & 05 & & $\mathrm{CO}$ \\
\hline 1283.3067 & 3.5 & 2.7 & 75.5 & 126 & $\mathrm{C} 85$ & H35 & N14 & 02 & & \\
\hline 1283.3067 & 3.5 & 2.7 & 78.0 & 83 & $\mathrm{C} 97$ & $\mathrm{H} 42$ & N3 & $\mathrm{Cl}$ & \multirow[b]{2}{*}{$\mathrm{CO}$} & \\
\hline 1283.3066 & 3.6 & 2.8 & 58.0 & 130 & $\mathrm{C} 81$ & H5 2 & N4 & 09 & & \\
\hline 1283.3066 & 3.6 & 2.8 & 63.5 & 134 & $\mathrm{C} 8 \mathrm{O}$ & $\mathrm{H} 46$ & N11 & 04 & $\mathrm{CO}$ & \\
\hline 1283.3066 & 3.6 & 2.8 & 66.0 & 106 & $\mathrm{C} 92$ & H5 3 & $\mathrm{O} 2$ & $\mathrm{Cl}$ & $\mathrm{Co}$ & \\
\hline 1283.3138 & -3.6 & -2.8 & 62.0 & 104 & $\mathrm{C} 86$ & H5 3 & N4 & 03 & $\mathrm{Cl}$ & $\mathrm{CO}$ \\
\hline 1283.3138 & -3.6 & -2.8 & 59.5 & 138 & C74 & $\mathrm{H} 46$ & N15 & 05 & $\mathrm{CO}$ & \\
\hline 1283.3138 & -3.6 & -2.8 & 54.0 & 137 & C75 & $\mathrm{H} 52$ & N8 & 010 & $\mathrm{CO}$ & \\
\hline 1283.3139 & -3.7 & -2.9 & 74.0 & 88 & $\mathrm{C} 91$ & $\mathrm{H} 42$ & N7 & 0 & $\mathrm{Cl}$ & \\
\hline
\end{tabular}




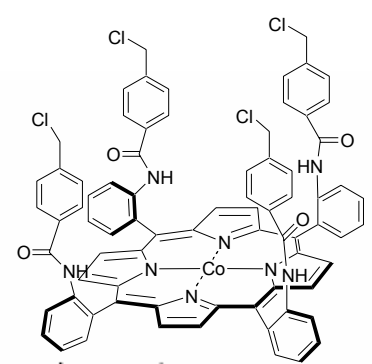

Single Mass Analysis

Tolerance $=5.0$ PPM / DBE: $\min =-1.5, \max =120.0$

Isotope cluster parameters: Separation $=1.0$ Abundance $=1.0 \%$

Monoisotopic Mass, Odd and Even Electron lons

17648 formula(e) evaluated with 244 results within limits (up to 140 closest results for each mass)

Yilong Yan, Yan-4-14

Cone $\mathrm{V}=30$

Coll $\vee=10.0$

YANY 051102_16244_YAN-4-14_HRMS 75 (2.564) Cn (Cen,6, 80.00, Ar); Sm (SG, 2x3.00); Sb (2,20.00); Cm (64:75)

02-Nov-2005

10:58:49

TOF MS ES+

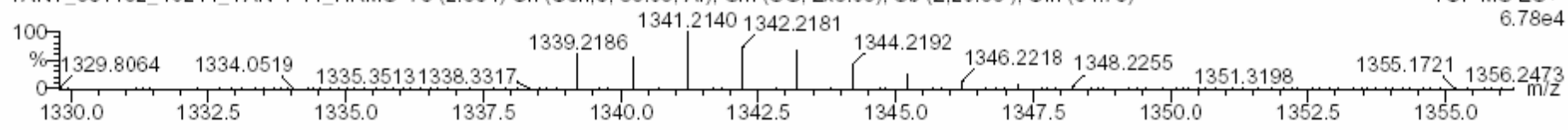

M1nimum :

Maximum :

$-1.5$

Calc. Mass

PPM

DBE

score

Formula

1339.2186

0.1

0.0

1339.2185

0.1

0.0

60.0

16

0.1

1339.2184

1339.2184

1339.2184

1339.2188

1339.2184

1339.2188

1339.2183

1339.2183

1339.2189

1339.2189

1339.2183

1339.2181

1339.2181

1339.2191

1339.2192

1339.2180

1339.2179

1339.2179

1339.2179

1339.2193

1339.2193

1339.2179

1339.2193

1339.2193

1339.2193

1339.2178

1339.2178

1339.2194

1339.2194

1339.2178

1339.2194

1339.2176

1339.2176

1339.2196

1339.2196

1339.2175

1339.2197

1339.2174

1339.2174

1339.2198

1339.2198

1339.2198

1339.2198

1339.2199

1339.2199

1339.2199

1339.2172

1339.2172

1339.2171

1339.2171

0.2

0.2

0.1

0.1

0.1

45.5

73.0

48.0

17

56

0.2

$-0.2$

0.2

0.1

$-0.1$

$-0.2$

0.1

0.3

$-0.1$

0.

0.2

-0.2
-0.2

$-0.3$

0.2

0.4

0.5

$-0.5$

$-0.6$

0.6

0.7

0.7

0.7

$-0.7$

$-0.7$

$-0.7$

$-0.7$

$-0.7$

0.8

0.8

$-0.8$

$-0.8$

0.8

$-0.8$

1.0

1.0
-1.0

$-1.0$

1.1

$-1.1$

1.2

78.5

53.5

67.5

84.0

62.0

66.5

72.0

74.0

68.5

74.5

64.5

$0.4 \quad 70.0$

$-0.4 \quad 76.0$

$\begin{array}{ll}-0.4 & 70.5\end{array}$

0.4

0.5

0.5

0.5

$-0.5$

$-0.5$

0.5

$-0.5$

$-0.5$

$-0.5$

0.6

0.6

$-0.6$

$-0.6$

0.6

$-0.6$

0.8

0.8

$-0.8$

$-0.8$

0.8

$-0.8$

0.9

0.9

$-1.2$

$-1.2$

$-0.9$

$-0.9$

$-0.9$

$-0.9$

$-1.0$

$-1.0$

$-1.0$

1.0

1. 0

1. 1

1. 1

72.5

52.5

83.0

58.0

63.0

88.0

88.5

57.5

82.5

52.0

71.0

76.5

69.5

64.0

79.0

61.5

69.0

74.5

71.5

66.0

77.0

63.5

57.0

62.5

83.5

53.0

78.0

47.5

65.0

59.5

57.0

1. 4

55.0

60.5

73.5

1. 5

48.5

1. 1

79.0

6

95

58

94

76
107

106

66

64

59

37
38

132

124

26

48

119

50

49
126

47

117

45

130

136

36

34

88
85

67

68

104

105

27

140

77

79

92
8

93

7

19

18
55

55
13

13
15
98

97

$\begin{array}{lllll}\mathrm{C} 80 & \mathrm{H} 45 & \mathrm{~N} 7 & \mathrm{O} 6 & \mathrm{Cl} 4\end{array}$

$\begin{array}{lllll}\mathrm{C} 79 & \mathrm{H} 39 & \mathrm{~N} 14 & \mathrm{O} & \mathrm{Cl} 4\end{array}$

$\begin{array}{llllll}\text { C63 } & \mathrm{H} 49 & \mathrm{~N} 15 & 010 & \mathrm{Cl} 3 & \mathrm{CO}\end{array}$

$\mathrm{C} 91 \mathrm{H} 38 \mathrm{~N} \quad 010 \mathrm{Cl}$

$\begin{array}{llllll}\mathrm{C} 75 & \mathrm{H} 56 & \mathrm{~N} 4 & \mathrm{O} & \mathrm{Cl} 4 & \mathrm{CO}\end{array}$

C90 H32 N8 O5 Cl

$\begin{array}{llllll}\mathrm{C} 74 & \mathrm{H} 50 & \mathrm{~N} 11 & \mathrm{O} 3 & \mathrm{Cl} 4 & \mathrm{CO}\end{array}$

$\mathrm{C} 81 \mathrm{H} 40 \mathrm{~N} 13$ O $\mathrm{Cl} 2 \mathrm{CO}$

$\begin{array}{llll}\mathrm{C} 89 & \mathrm{H} 26 & \mathrm{~N} 15 \mathrm{Cl}\end{array}$

$\begin{array}{llllll}\mathrm{C} 82 & \mathrm{H} 46 & \mathrm{~N} 6 & \mathrm{O} 6 & \mathrm{Cl} 2 & \mathrm{CO}\end{array}$

$\begin{array}{llllll}\mathrm{C} 85 & \mathrm{H} 43 & \mathrm{~N} 5 & \mathrm{O} & \mathrm{Cl} & \mathrm{CO}\end{array}$

$\begin{array}{llllll}\mathrm{C} 84 & \mathrm{H} 37 & \mathrm{~N} 12 & \mathrm{O} 2 & \mathrm{Cl} & \mathrm{CO}\end{array}$

$\begin{array}{lllll}\mathrm{C} 87 & \mathrm{H} 35 & \mathrm{~N} 9 & \mathrm{O} 4 & \mathrm{Cl} 2\end{array}$

$\begin{array}{lllll}\mathrm{C} 88 & \mathrm{H} 41 & \mathrm{~N} 2 & \mathrm{O} & \mathrm{Cl} 2\end{array}$

C96 $\mathrm{H} 44 \quad \mathrm{~N} \quad \mathrm{Cl} 2 \mathrm{CO}$

$\begin{array}{lllll}\mathrm{C} 83 & \mathrm{H} 42 & \mathrm{~N} 6 & \mathrm{O} 7 & \mathrm{Cl} 3\end{array}$

$\begin{array}{lllll}\mathrm{C} 82 & \mathrm{H} 36 & \mathrm{~N} 13 & \mathrm{O} 2 & \mathrm{Cl} 3\end{array}$

$\mathrm{C} 89 \mathrm{H} 36$ N8 04 Co

C9O H42 N 09 CO

$\mathrm{C} 94 \quad \mathrm{H} 43$ N2 $\mathrm{Cl} 4$ 


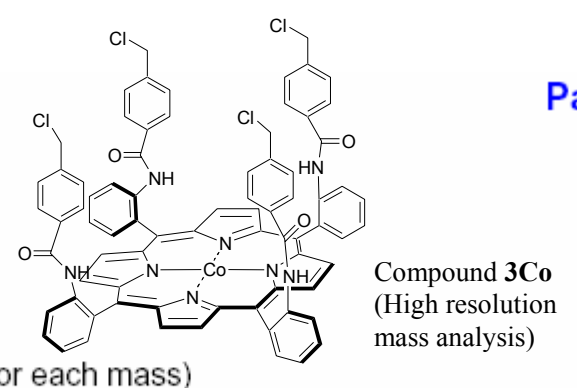

Single Mass Analysis

Tolerance $=5.0$ PPM / DBE: $\min =-1.5, \max =120.0$

Isotope cluster parameters: Separation $=1.0$ Abundance $=1.0 \%$

Monoisotopic Mass, Odd and Even Electron Ions

17648 formula(e) evaluated with 244 results within limits (up to 140 closest results for each mass)

$\begin{array}{ll}\text { Yilong Yan, Yan-4-14 } & \text { Cone } V=30 \\ 16129 & \text { Coll } V=10.0\end{array}$

YANY_051102_16244_YAN-4-14_HRMS 75 (2.564) Cn (Cen,6, 80.00, Ar); Sm (SG, 2x3.00); Sb (2,20.00); Cm (64:75)

02-Nov-2005

$10: 58: 49$

TOF MS ES+

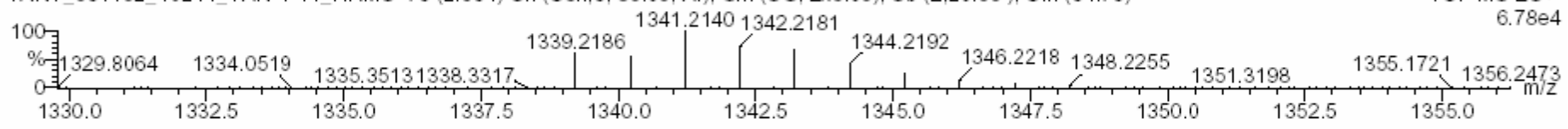

M1nimum :

Maximum :

Mass

\begin{tabular}{|c|c|c|c|c|}
\hline Calc. Mass & $\mathrm{mDa}$ & PPM & DBE & Scor \\
\hline 1339.2171 & 1.5 & 1.1 & 54.0 & 1 \\
\hline 1339.2201 & -1.5 & -1.1 & 67.0 & 75 \\
\hline 1339.2201 & -1.5 & -1.1 & 61.5 & 74 \\
\hline 1339.2171 & 1.5 & 1.1 & 81.5 & 57 \\
\hline 1339.2201 & -1.5 & -1.2 & 59.0 & 110 \\
\hline 1339.2170 & 1.6 & 1.2 & 67.0 & 109 \\
\hline 1339.2202 & -1.6 & -1.2 & 79.0 & 65 \\
\hline 1339.2170 & 1.6 & 1.2 & 72.5 & 108 \\
\hline 1339.2202 & -1.6 & -1.2 & 73.5 & 63 \\
\hline 1339.2203 & -1.7 & -1.2 & 40.5 & 23 \\
\hline 1339.2167 & 1.9 & 1.4 & 65.0 & 40 \\
\hline 1339.2205 & -1.9 & -1.4 & 81.0 & 134 \\
\hline 1339.2205 & -1.9 & -1.4 & 75.5 & 127 \\
\hline 1339.2166 & 2.0 & 1.5 & 78.0 & 116 \\
\hline 1339.2166 & 2.0 & 1.5 & 53.0 & 51 \\
\hline 1339.2166 & 2.0 & 1.5 & 83.5 & 125 \\
\hline 1339.2166 & 2.0 & 1.5 & 58.5 & 52 \\
\hline 1339.2206 & -2.0 & -1.5 & 62.5 & 46 \\
\hline 1339.2206 & -2.0 & -1.5 & 87.5 & 121 \\
\hline 1339.2206 & -2.0 & -1.5 & 57.0 & 44 \\
\hline 1339.2206 & -2.0 & -1.5 & 82.0 & 115 \\
\hline 1339.2166 & 2.0 & 1.5 & 61.0 & 24 \\
\hline 1339.2206 & -2.0 & -1.5 & 54.5 & 87 \\
\hline 1339.2165 & 2.1 & 1.6 & 71.5 & 133 \\
\hline 1339.2165 & 2.1 & 1.6 & 77.0 & 138 \\
\hline 1339.2207 & -2.1 & -1.6 & 69.0 & 33 \\
\hline 1339.2207 & -2.1 & -1.6 & 63.5 & 30 \\
\hline 1339.2164 & 2.2 & 1.6 & 79.5 & 89 \\
\hline 1339.2162 & 2.4 & 1.8 & 69.5 & 69 \\
\hline 1339.2162 & 2.4 & 1.8 & 75.0 & 71 \\
\hline 1339.2210 & -2.4 & -1.8 & 71.0 & 103 \\
\hline 1339.2162 & 2.4 & 1.8 & 77.5 & 29 \\
\hline 1339.2161 & 2.5 & 1.9 & 57.5 & 80 \\
\hline 1339.2161 & 2.5 & 1.9 & 63.0 & 82 \\
\hline 1339.2211 & -2.5 & -1.9 & 58.0 & 11 \\
\hline 1339.2211 & -2.5 & -1.9 & 83.0 & 90 \\
\hline 1339.2211 & -2.5 & -1.9 & 52.5 & 9 \\
\hline 1339.2211 & -2.5 & -1.9 & 77.5 & 91 \\
\hline 1339.2161 & 2.5 & 1.9 & 65.5 & 32 \\
\hline 1339.2211 & -2.5 & -1.9 & 75.0 & 135 \\
\hline 1339.2212 & -2.6 & -2.0 & 64.5 & 21 \\
\hline 1339.2212 & -2.6 & -2.0 & 59.0 & 20 \\
\hline 1339.2160 & 2.6 & 2.0 & 84.0 & 122 \\
\hline 1339.2212 & -2.6 & -2.0 & 56.5 & 53 \\
\hline 1339.2159 & 2.7 & 2.0 & 55.5 & 10 \\
\hline 1339.2159 & 2.7 & 2.0 & 61.0 & 12 \\
\hline 1339.2157 & 2.9 & 2.1 & 74.0 & 102 \\
\hline 1339.2157 & 2.9 & 2.1 & 49.0 & 2 \\
\hline 1339.2157 & 2.9 & 2.1 & 79.5 & 101 \\
\hline 1339.2215 & -2.9 & -2.1 & 66.5 & 72 \\
\hline 1339.2215 & -2.9 & -2.1 & 61.0 & 70 \\
\hline 1339.2157 & 2.9 & 2.2 & 82.0 & 58 \\
\hline 1339.2215 & -2.9 & -2.2 & 58.5 & 111 \\
\hline 1339.2156 & 3.0 & 2.2 & 62.0 & 113 \\
\hline 1339.2156 & 3.0 & 2.2 & 67.5 & 112 \\
\hline 1339.2216 & -3.0 & -2.2 & 78.5 & 62 \\
\hline
\end{tabular}

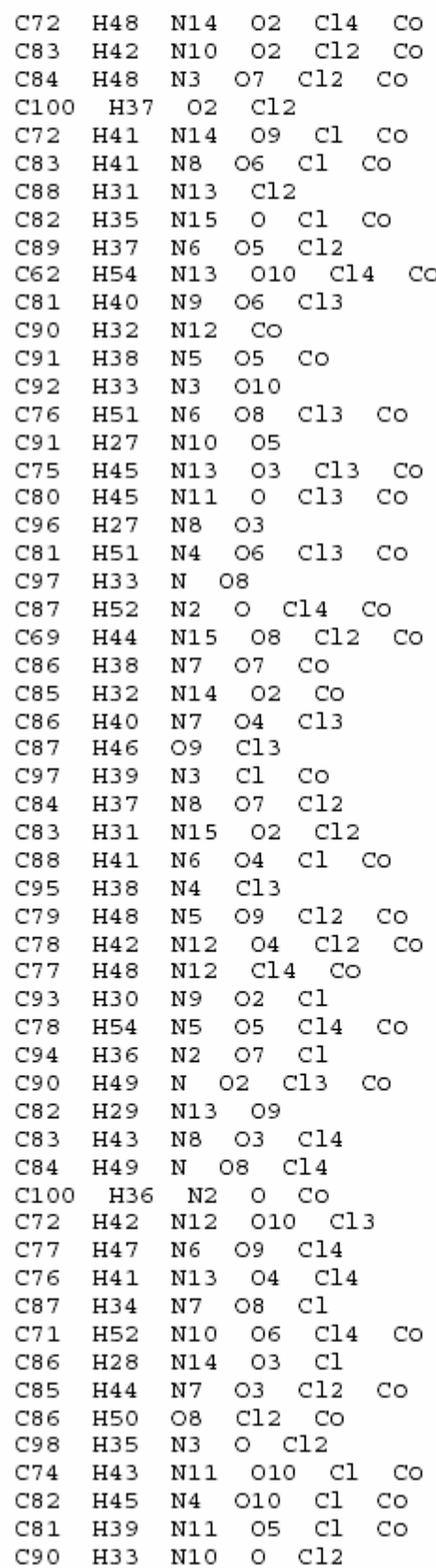




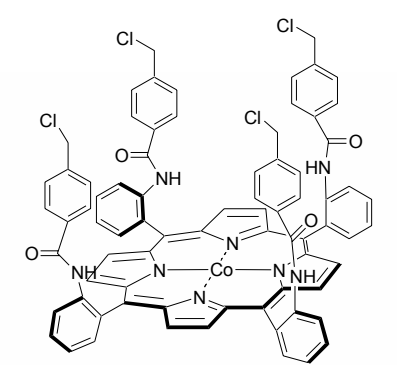

$=\mathrm{O}$

\section{Single Mass Analysis}

Tolerance $=5.0$ PPM / DBE: $\min =-1.5, \max =120.0$

Isotope cluster parameters: Separation $=1.0$ Abundance $=1.0 \%$

Monoisotopic Mass, Odd and Even Electron Ions

17648 formula(e) evaluated with 244 results within limits (up to 140 closest results for each mass) $\begin{array}{ll}\text { Yilong Yan, Yan-4-14 } & \text { Cone } V=30 \\ 16129 & \text { Coll } V=10.0\end{array}$

YANY_051102_16244_YAN-4-14_HRMS 75 (2.564) Cn (Cen,6, 80.00, Ar); Sm (SG, 2x3.00); Sb (2,20.00); Cm (64:75)
02-Nov-2005 $10: 58: 49$ TOF MS ES+
Compound $\mathbf{3 C o}$ mass analysis)

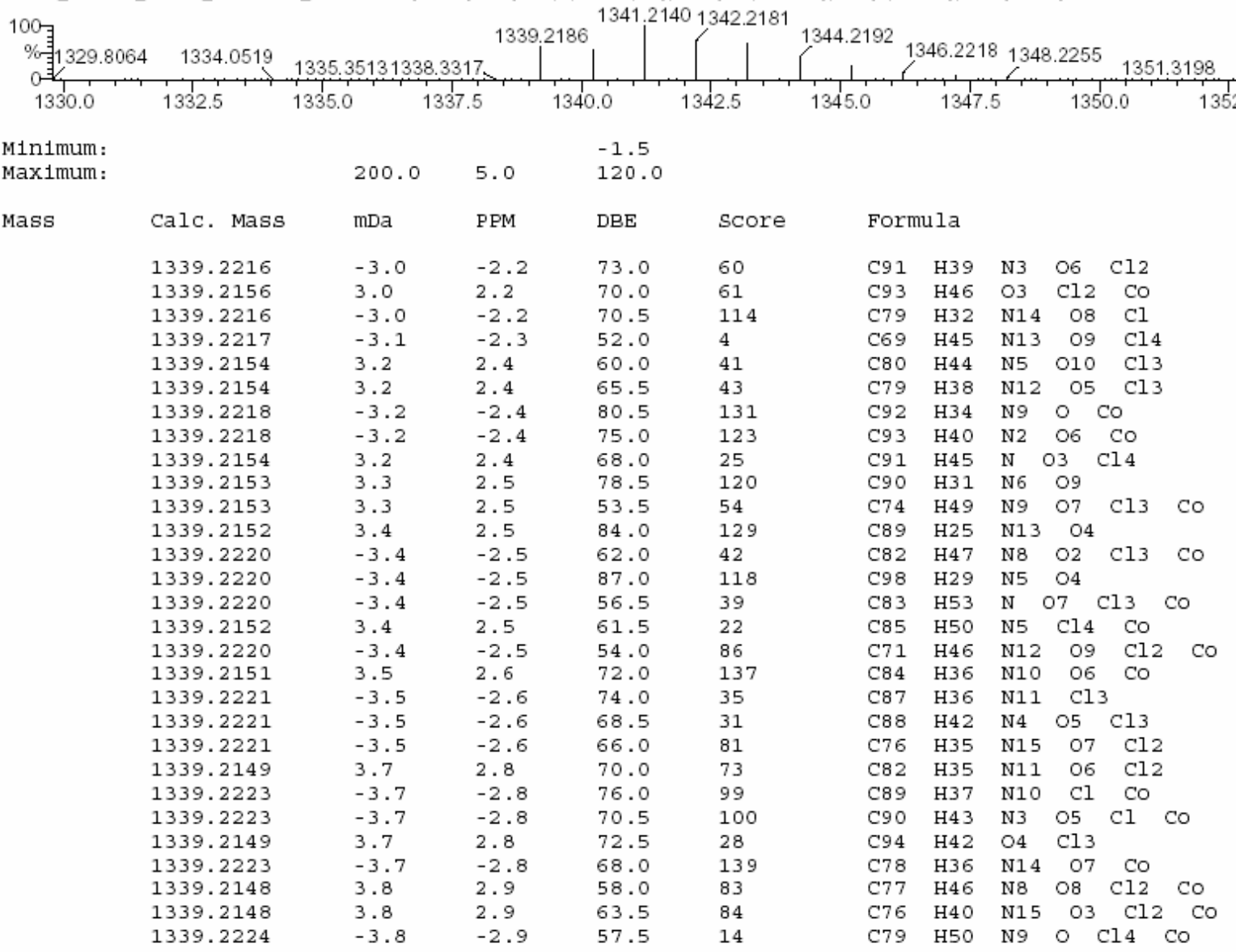




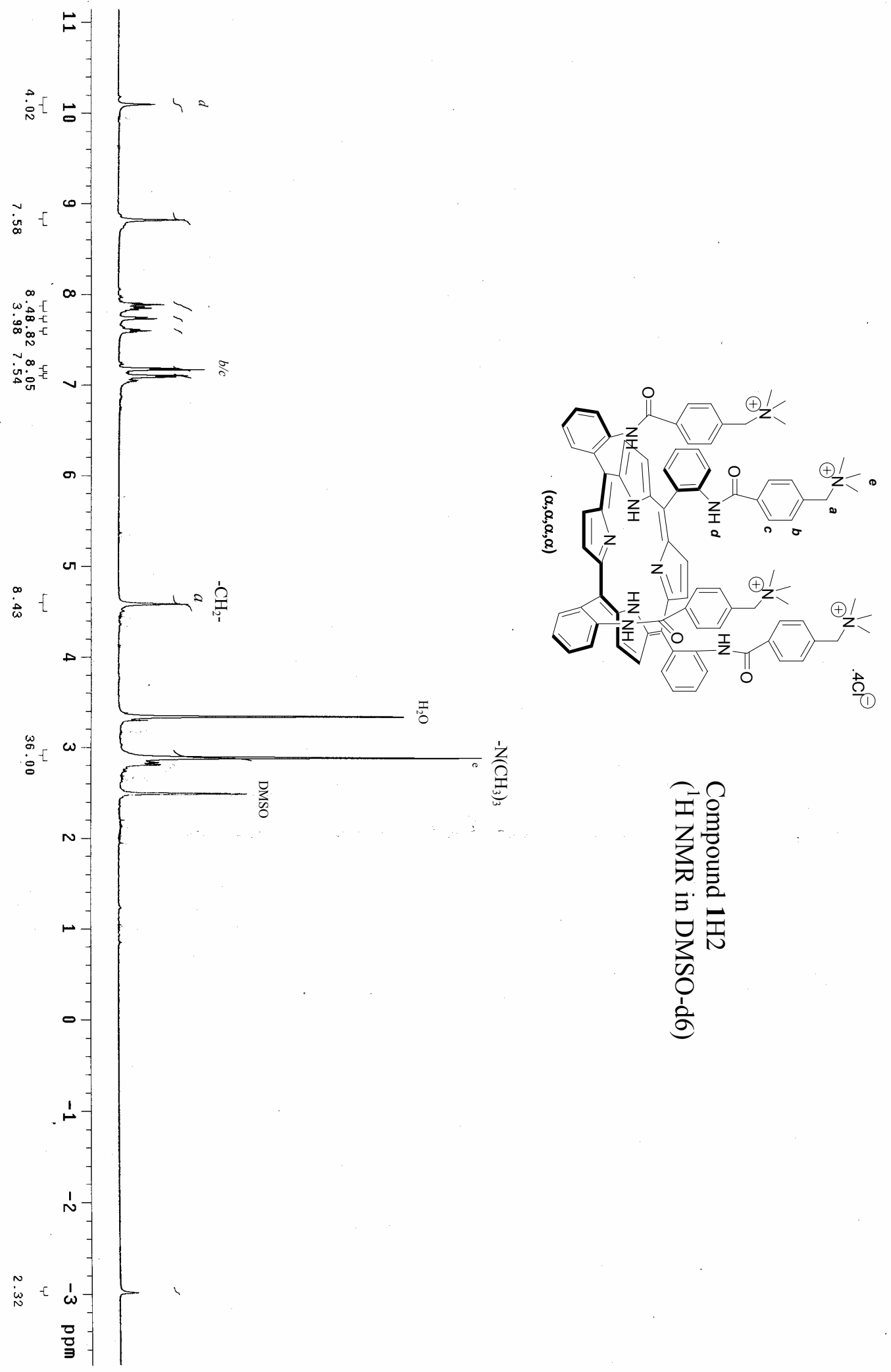




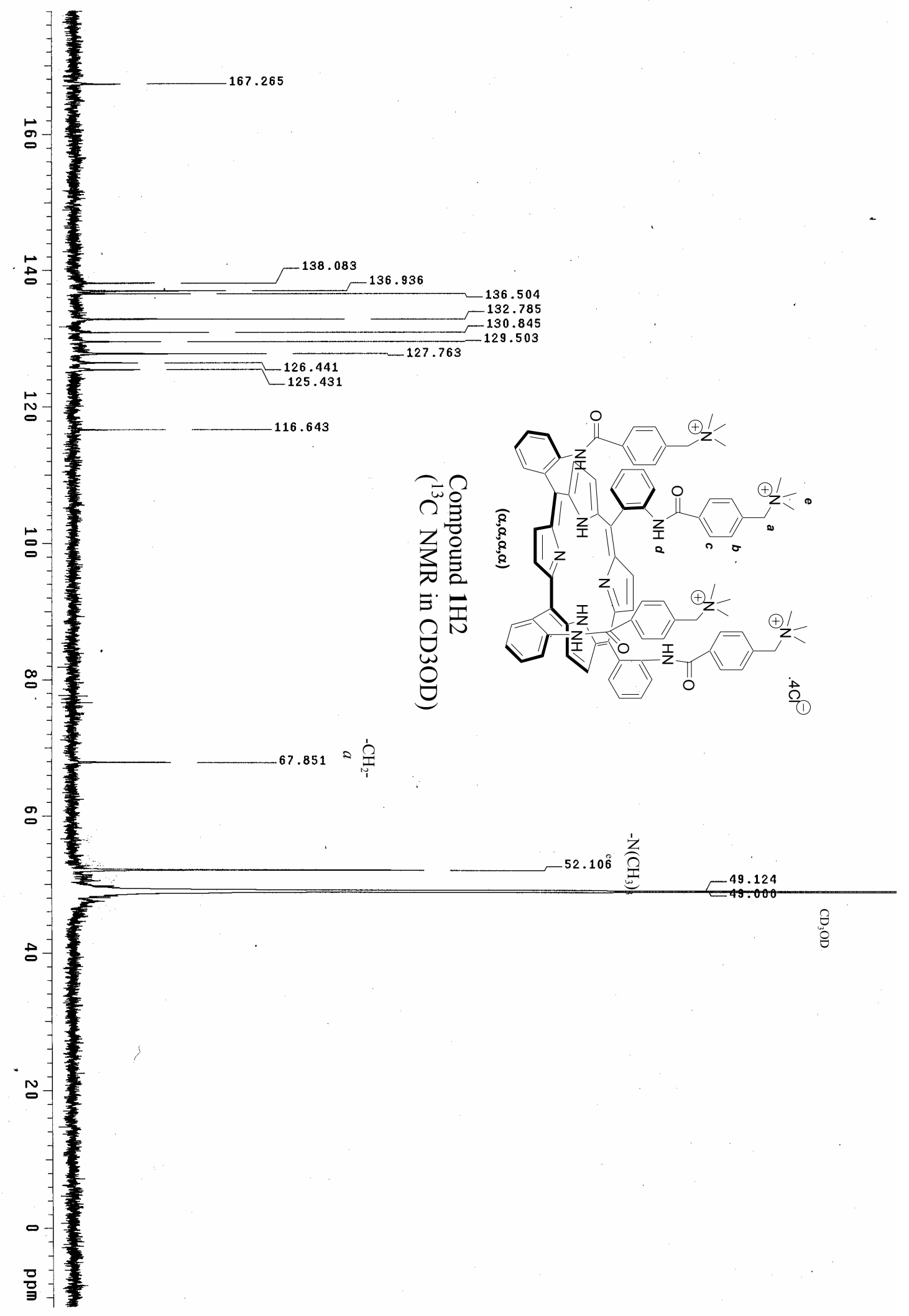




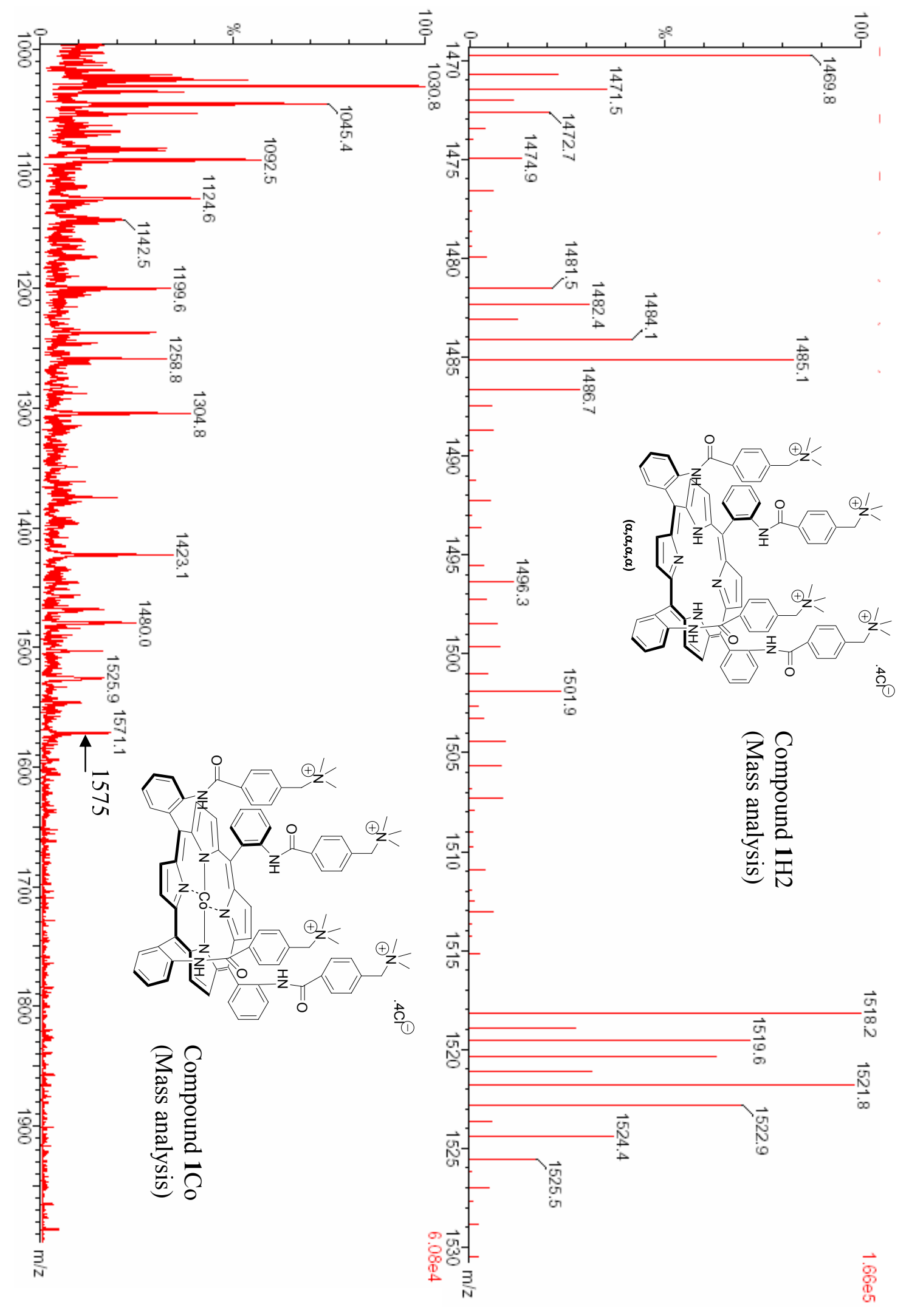




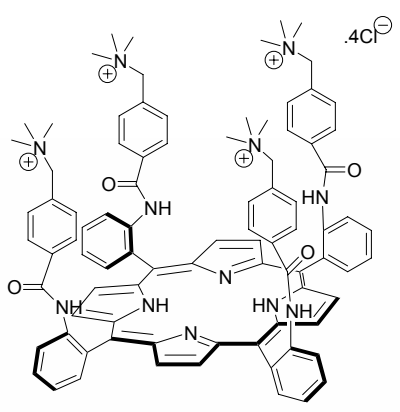

Single Mass Analysis

Tolerance $=5.0$ PPM / DBE: $\min =-1.5, \max =120.0$

Isotope cluster parameters: Separation $=1.0$ Abundance $=1.0 \%$

Monoisotopic Mass, Odd and Even Electron Ions

5740 formula(e) evaluated with 103 results within limits (up to 140 closest results for each mass)

YANY_051102_16244_YAN-1-60_HRMS 63 (2.155) Cn (Cen,6, 80.00, Ar); Sm (SG, 2×3.00); Sb (2,20.00); Cm (54:63)

$\begin{array}{rrr}1484.6273 & 1485.6251 & 1486.6272\end{array}$

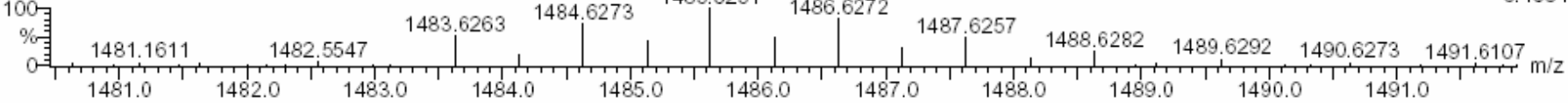

Minimum :

Maximum :

Mass

1483.6263
Calc. Mass

1483.6264

1483.6265

1483.6260

1483.6260

1483.6260

1483.6259

1483.6269

1483.6269

1483.6255

1483.6255

1483.6272

1483.6272

1483.6273

1483.6274

1483.6274

1483.6252

1483.6250

1483.6250

1483.6277

1483.6277

1483.6278

1483.6279

1483.6247

1483.6247

1483.6247

1483.6246

1483.6282

1483.6282

1483.6242

1483.6242

1483.6286

1483.6287

1483.6287

1483.6238

1483.6238

1483.6237

1483.6291

1483.6291

1483.6291

1483.6292

1483.6292

1483.6233

1483.6233

1483.6232

1483.6232

1483.6296

1483.6296

1483.6297

1483.6228

1483.6228

1483.6228

1483.6300

1483.6300

1483.6225

1483.6224

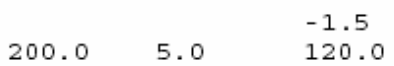

$\mathrm{mDa} \quad \mathrm{PPM}$

$-0.1 \quad-0.1$

57.5

57.5
39.0

43.5

49.0

51.5

62.0

53.0

47.5

48.0

53.5

67.0

61.5

51.0

48.5

43.0

39.5

52.5

58.0

62.5

57.0

44.0

38.5

44.0

46.5

52.0

62.5

58.0

52.5

48.5

56.5

66.5

48.0

42.5

34.5

40.0

53.0

62.0

56.5

54.0

43.5

38.0

44.5

47.0

57.5

63.0

57.5

52.0

31.0

49.0

51.5

57.0

53.0

47.5

35.0

53.5

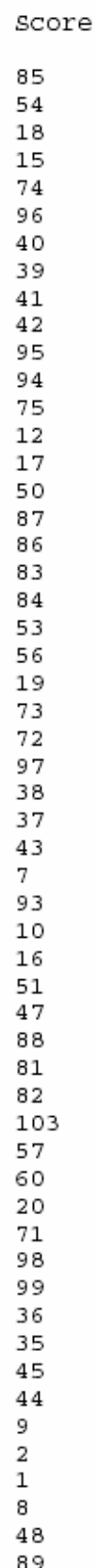

Formula

$\begin{array}{llllr}\mathrm{C} 94 & \mathrm{H} 84 & \mathrm{~N} 10 & \mathrm{O} 6 & \mathrm{Cl} \\ \mathrm{C} 84 & \mathrm{H} 97 & \mathrm{~N} 9 & \mathrm{O} & \mathrm{Cl} 4\end{array}$

$\begin{array}{lllll}\mathrm{C} 84 & \mathrm{H} 97 & \mathrm{~N} 9 & 07 & \mathrm{Cl} 4 \\ \mathrm{C} 87 & \mathrm{H} 94 & \mathrm{~N} 8 & 08 & \mathrm{Cl} 3\end{array}$

$\begin{array}{lllll}\mathrm{C} 86 & \mathrm{H} 88 & \mathrm{~N} 15 & \mathrm{O} 3 & \mathrm{Cl} 3\end{array}$

$\begin{array}{lllll}\mathrm{C} 98 & \mathrm{H} 95 & \mathrm{~N} 4 & \mathrm{Cl} & \mathrm{Cl}\end{array}$

$\begin{array}{llll}\mathrm{C} 97 & \mathrm{H} 81 & \text { N9 } & \text { O7 }\end{array}$

$\begin{array}{lllll}\mathrm{C} 91 & \mathrm{H} 87 & \mathrm{~N} 11 & 05 & \mathrm{Cl} 2\end{array}$

$\begin{array}{lllll}\mathrm{C} 92 & \mathrm{H} 93 & \mathrm{~N} 4 & 010 & \mathrm{Cl} 2\end{array}$

$\begin{array}{lllll}\mathrm{C} 90 & \mathrm{H} 91 & \mathrm{~N} 7 & \mathrm{O} & \mathrm{Cl} 2\end{array}$

$\begin{array}{lllll}\mathrm{C} 89 & \mathrm{H} 85 & \mathrm{~N} 14 & \mathrm{O} 4 & \mathrm{Cl} 2\end{array}$

$\begin{array}{llll}\mathrm{C} 98 & \mathrm{H} 77 & \mathrm{~N} 13 & \mathrm{O} 3\end{array}$

$\begin{array}{lcccc}\mathrm{C} 99 & \mathrm{H} 83 & \mathrm{~N} 6 & 08 & \\ \mathrm{C} 100 & \mathrm{H} 97 & \mathrm{~N} & 02 & \mathrm{Cl} 4\end{array}$

$\begin{array}{lllll}\mathrm{C} 88 & \mathrm{H} 90 & \mathrm{~N} 12 & \mathrm{O} 4 & \mathrm{Cl} 3\end{array}$

$\begin{array}{lllll}\mathrm{C} 89 & \mathrm{H} 96 & \mathrm{~N} 5 & 09 & \mathrm{Cl} 3\end{array}$

$\begin{array}{lllll}\mathrm{C} 82 & \mathrm{H} 95 & \mathrm{~N} 12 & 06 & \mathrm{Cl} 4\end{array}$

C93 H88 N6 O10 Cl

$\begin{array}{lllll}\mathrm{C} 92 & \mathrm{H} 82 & \mathrm{~N} 13 & 05 & \mathrm{Cl}\end{array}$

$\begin{array}{lllll}\mathrm{C} 95 & \mathrm{H} 80 & \mathrm{~N} 14 & \mathrm{O} 2 & \mathrm{Cl}\end{array}$

$\begin{array}{lllll}\mathrm{C} 96 & \mathrm{H} 86 & \mathrm{~N} 7 & 07 & \mathrm{Cl}\end{array}$

$\begin{array}{lllll}\mathrm{C} 85 & \mathrm{H} 93 & \mathrm{~N} 13 & \mathrm{O} 3 & \mathrm{Cl} 4\end{array}$

$\begin{array}{lllll}\mathrm{C} 86 & \mathrm{H} 99 & \mathrm{~N} 6 & 08 & \mathrm{Cl} 4\end{array}$

$\begin{array}{lllll}\mathrm{C} 85 & \mathrm{H} 92 & \mathrm{~N} 11 & 07 & \mathrm{Cl} 3\end{array}$

$\begin{array}{llll}\mathrm{C} 97 & \mathrm{H} 99 & 05 & \mathrm{Cl} 4\end{array}$

$\begin{array}{llll}\mathrm{C} 96 & \mathrm{H} 93 & \mathrm{~N} 7 & \mathrm{Cl} 4\end{array}$

C95 H79 N12 O6

$\begin{array}{lllll}\mathrm{C} 92 & \mathrm{H} 83 & \mathrm{~N} 15 & \mathrm{O} & \mathrm{Cl} 2\end{array}$

$\begin{array}{lllll}\mathrm{C} 93 & \mathrm{H} 89 & \mathrm{~N} 8 & \mathrm{C} & \mathrm{Cl}\end{array}$

$\begin{array}{lllll}\mathrm{C} 88 & \mathrm{H} 89 & \mathrm{~N} 10 & 08 & \mathrm{Cl} 2\end{array}$

$\begin{array}{lllll}\mathrm{C} 99 & \mathrm{H} 90 & \mathrm{~N} 6 & \mathrm{Cl}\end{array}$

$\begin{array}{llll}\mathrm{C} 100 & \mathrm{H} 79 & \mathrm{~N} 10 & \mathrm{O}\end{array}$

$\begin{array}{lllll}\mathrm{C} 90 & \mathrm{H} 92 & \mathrm{~N} 9 & \mathrm{C} & \mathrm{Cl} 3\end{array}$

$\begin{array}{lllll}\mathrm{C} 91 & \mathrm{H} 98 & \mathrm{~N} 2 & 010 & \mathrm{Cl} 3\end{array}$

$\begin{array}{lllll}\mathrm{C} 81 & \mathrm{H} 99 & \mathrm{~N} 8 & 010 & \mathrm{Cl} 4\end{array}$

$\begin{array}{lllll}\mathrm{C} 80 & \mathrm{H} 93 & \mathrm{~N} 15 & \mathrm{O} & \mathrm{Cl} 4\end{array}$

C91 H86 N9 O9 Cl

$\begin{array}{lllll}\mathrm{C} 97 & \mathrm{H} 82 & \mathrm{~N} 11 & 03 & \mathrm{Cl}\end{array}$

$\begin{array}{lllll}\mathrm{C} 98 & \mathrm{H} 88 & \mathrm{~N} 4 & \mathrm{O} 8 & \mathrm{Cl}\end{array}$

$\begin{array}{llll}\mathrm{C} 86 & \mathrm{H} 81 & \mathrm{~N} 15 & 010\end{array}$

$\begin{array}{lllll}\mathrm{C} 87 & \mathrm{H} 95 & \mathrm{~N} 10 & \mathrm{O} 4 & \mathrm{Cl} 4\end{array}$

$\begin{array}{lllll}\mathrm{C} 88 & \mathrm{H} 101 & \mathrm{~N} 3 & 09 & \mathrm{Cl} 4\end{array}$

$\begin{array}{lllll}\mathrm{C} 83 & \mathrm{H} 90 & \mathrm{~N} 14 & \mathrm{O} & \mathrm{Cl} 3\end{array}$

$\begin{array}{lllll}\mathrm{C} 95 & \mathrm{H} 97 & \mathrm{~N} 3 & \mathrm{O} & \mathrm{Cl}\end{array}$

C94 H83 N8 O10

$\begin{array}{llll}\mathrm{C} 93 & \mathrm{H} 77 & \mathrm{~N} 15 & \mathrm{O}\end{array}$

$\begin{array}{lllll}\mathrm{C} 94 & \mathrm{H} 85 & \mathrm{~N} 12 & \mathrm{O} 2 & \mathrm{Cl} 2\end{array}$

$\begin{array}{lllll}\mathrm{C} 95 & \mathrm{H} 91 & \mathrm{~N} 5 & \mathrm{O} & \mathrm{Cl} 2\end{array}$

$\begin{array}{lllll}\mathrm{C} 73 & \mathrm{H} 97 & \mathrm{~N} 15 & 010 & \mathrm{Cl} 4\end{array}$

$\begin{array}{lllll}\mathrm{C} 86 & \mathrm{H} 87 & \mathrm{~N} 13 & 07 & \mathrm{Cl} 2\end{array}$

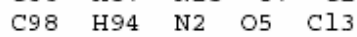

C97 H88 N9 Cl3

$\begin{array}{lllll}\mathrm{C} 91 & \mathrm{H} 88 & \mathrm{~N} 13 & \mathrm{O} & \mathrm{Cl} 3\end{array}$

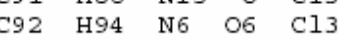

$\begin{array}{lllll}\mathrm{C} 79 & \mathrm{H} 97 & \mathrm{~N} 11 & 09 & \mathrm{Cl} 4 \\ \mathrm{C} 89 & \mathrm{H} 84 & \mathrm{~N} 12 & 08 & \mathrm{Cl}\end{array}$
Compound $\mathbf{1 H} 2$

(High resolution

Mass analysis) 1491.0 


\section{Elemental Composition Report}

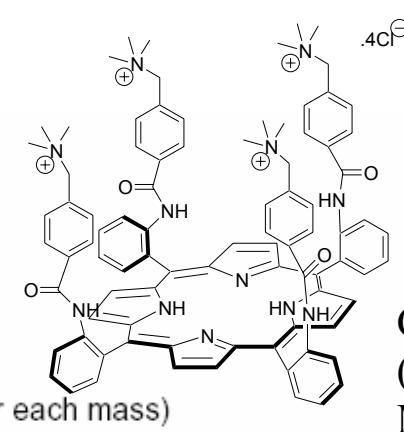

Single Mass Analysis

Tolerance $=5.0$ PPM / DBE: $\min =-1.5, \max =120.0$

Isotope cluster parameters: Separation $=1.0$ Abundance $=1.0 \%$

Monoisotopic Mass, Odd and Even Electron Ions

5740 formula(e) evaluated with 103 results within limits (up to 140 closest results for each mass)

Page 2

Cone $\mathrm{V}=15$

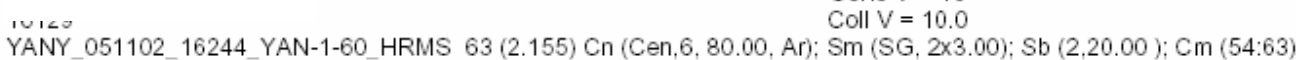

$\begin{array}{lllll}100 & 1484.6273 & 1485.6251 & 1486.6272\end{array}$

$3.46 \mathrm{e} 4$

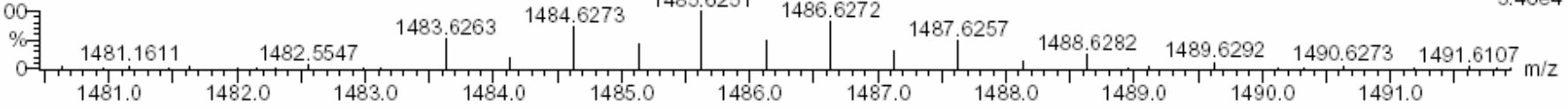

Minimum :

Maximum :

Mass

Calc. Mas

200.0

5.0

$-1.5$

mDa PPM

DBE

score

Formula

1483.6223

4.0

2. 7

61.5

1483.6304

$-4.1$

$-2.8$

61.5

1483.6305

$-4.1$

$-2.8$

56.0

$\begin{array}{ll}-4.2 & -2.8 \\ -4.2 & -2.9\end{array}$

48.5

1483.6305

-2 .

43.0

1483.6305

$-4.2$

$4.3 \quad 2.9$

1483.6220

1483.6219

1483.6309

1483.6309

1483.6309

1483.6215

1483.6314

1483.6314

1483.6314

1483.6211

1483.6210

1483.6210

1483.6210

1483.6317

1483.6319

1483.6319

1483.6319

1483.6207

1483.6206

1483.6206

1483.6205

1483.6322

1483.6202

1483.6202

1483.6201

1483.6327

1483.6327

1483.6327

1483.6197

1483.6197

1483.6331

1483.6332

1483.6332

1483.6332

1483.6193

$4.3 \quad 2.9$

39.5

$-4.6 \quad-3.1$

$-4.6 \quad-3.1$

$-4.6 \quad-3.1$

4.82

$\begin{array}{ll}-5.1 & -3.4 \\ -5.1 & -3.4\end{array}$

$-5.1 \quad-3.4$

$5.2 \quad 3.5$

$5.3 \quad 3.6$

$5.3 \quad 3.6$

$5.3 \quad 3.6$

$-5.4 \quad-3.7$

$-5.6 \quad-3.8$

$-5.6 \quad-3.8$

$-5.6 \quad-3.8$

$\begin{array}{ll}5.6 & 3.8\end{array}$

$5.7 \quad 3.8$

$5.7 \quad 3.8$

$5.8 \quad 3.9$

$-5.9-4.0$

$6.1 \quad 4.1$

$6.2 \quad 4.1$

$-6.4 \quad-4.3$

$-6.4 \quad-4.3$

6.6

$\begin{array}{ll}6.6 & 4.5 \\ 6.6 & 4.5\end{array}$

$-6.8 \quad-4.6$

$-6.9 \quad-4.7$

$-6.9 \quad-4.7$

$\begin{array}{ll}-6.9 & -4.7\end{array}$

7.0

7.0

1483.6193

1483.6192

1483.6192

1483.6336

1483.6336

7.1

7.1

$-7.3$

4.7

4. 7

4. 8

4.8

$-4.9$

47.5

57.0
51.5

49.0

52.0

52.5

47.0

44.5

35.5

54.0

56.5

62.0

66.5

48.0

42.5

40.0

40.0

42.5

48.0

58.5

56.5

44.5

47.0

52.5

52.0

46.5

44.0

51.5

57.0

58.0

47.5

42.0

39.5

43.0

48.5

61.5

67.0

61.5

56.0

25

79

80
58

61

65

21
69

100

34

33
92

6

11
55

46

91
28

28
29

76

62

66
24

23

68

64

30

52

13

4
5

14

49

31
32

101

67

70

22
63

59

$-7.3 \quad-4.9$

$-4.9$

53.5

$\begin{array}{lllll}\mathrm{C} 100 & \mathrm{H} 85 & \mathrm{~N} 8 & \mathrm{Cl}\end{array}$

C100 H90 $\mathrm{N}$ O9 $\mathrm{Cl}$

$\begin{array}{llll}\mathrm{C} 88 & \mathrm{H} 91 & \mathrm{~N} 14 & \mathrm{Cl} 4\end{array}$

$\begin{array}{lllll}\mathrm{C} 89 & \mathrm{H} 97 & \mathrm{~N} 7 & \mathrm{O} & \mathrm{Cl} 4\end{array}$

$\begin{array}{llll}\mathrm{C} 90 & \mathrm{H} 103 & 010 & \mathrm{Cl} 4\end{array}$

$\begin{array}{lllll}\mathrm{C} 82 & \mathrm{H} 94 & \mathrm{~N} 10 & 010 & \mathrm{Cl} 3\end{array}$

$\begin{array}{lllll}\mathrm{C} 93 & \mathrm{H} 95 & \mathrm{~N} 6 & \mathrm{O} & \mathrm{Cl} 4\end{array}$

C92 H81 N11 O9

$\begin{array}{lllll}\mathrm{C} 96 & \mathrm{H} 87 & \mathrm{~N} 9 & \mathrm{O} 3 & \mathrm{Cl} 2\end{array}$

$\begin{array}{lllll}\mathrm{C} 97 & \mathrm{H} 93 & \mathrm{~N} 2 & \mathrm{O} 8 & \mathrm{Cl} 2\end{array}$

$\mathrm{C} 85 \mathrm{H} 86 \quad \mathrm{~N} 13 \quad 010 \mathrm{Cl}$

$\begin{array}{lllll}\mathrm{C} 96 & \mathrm{H} 92 & \mathrm{~N} 5 & \mathrm{O} & \mathrm{Cl} 3\end{array}$

$\begin{array}{lllll}\mathrm{C} 93 & \mathrm{H} 90 & \mathrm{~N} 10 & \mathrm{O} 2 & \mathrm{Cl} 3\end{array}$

$\begin{array}{lllll}\mathrm{C} 94 & \mathrm{H} 96 & \mathrm{~N} 3 & \mathrm{O} & \mathrm{Cl} 3\end{array}$

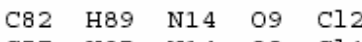

$\begin{array}{lllll}\mathrm{C} 77 & \mathrm{H} 95 & \mathrm{~N} 14 & 08 & \mathrm{Cl} 4\end{array}$

$\begin{array}{lllll}\mathrm{C} 87 & \mathrm{H} 82 & \mathrm{~N} 15 & 07 & \mathrm{Cl}\end{array}$

$\begin{array}{lllll}\mathrm{C} 99 & \mathrm{H} 89 & \mathrm{~N} 4 & \mathrm{O} & \mathrm{Cl} 2\end{array}$

$\begin{array}{llll}\mathrm{C} 98 & \mathrm{H} 83 & \mathrm{~N} 11 & \mathrm{Cl} 2\end{array}$

$\mathrm{C} 100 \mathrm{H} 80 \mathrm{~N} 12 \mathrm{Cl}$

$\begin{array}{lllll}\mathrm{C} 90 & \mathrm{H} 93 & \mathrm{~N} 11 & \mathrm{C} & \end{array}$

$\begin{array}{lllll}\mathrm{C} 91 & \mathrm{H} 99 & \mathrm{~N} 4 & \mathrm{O} & \mathrm{Cl}\end{array}$

$\begin{array}{lllll}\mathrm{C} 79 & \mathrm{H} 92 & \mathrm{~N} 15 & \mathrm{O} 8 & \mathrm{Cl} 3\end{array}$

$\begin{array}{lllll}\mathrm{C} 80 & \mathrm{H} 92 & \mathrm{~N} 13 & 09 & \mathrm{Cl} 3\end{array}$

$\begin{array}{lllll}\mathrm{C} 92 & \mathrm{H} 99 & \mathrm{~N} 2 & \mathrm{O} & \mathrm{Cl}\end{array}$

$\begin{array}{lllll}\mathrm{C} 91 & \mathrm{H} 93 & \mathrm{~N} 9 & \mathrm{O} 2 & \mathrm{Cl} 4\end{array}$

C90 H79 N14 O8

$\begin{array}{lllll}\mathrm{C} 98 & \mathrm{H} 89 & \mathrm{~N} 6 & \mathrm{O} 4 & \mathrm{Cl} 2\end{array}$

$\mathrm{C} 83 \mathrm{H} 89$ N12 $010 \quad \mathrm{Cl} 2$

$\begin{array}{lllll}\mathrm{C} 95 & \mathrm{H} 96 & \mathrm{~N} & \mathrm{O} 8 \mathrm{Cl} 3\end{array}$

$\begin{array}{lllll}\mathrm{C} 94 & \mathrm{H} 90 & \mathrm{~N} 8 & \mathrm{O} & \mathrm{Cl} 3\end{array}$

$\begin{array}{lllll}\mathrm{C} 95 & \mathrm{H} 92 & \mathrm{~N} 7 & \mathrm{O} & \mathrm{Cl}\end{array}$

$\begin{array}{llll}\mathrm{C} 96 & \mathrm{H} 98 & 08 & \mathrm{Cl} 3\end{array}$

$\begin{array}{lllll}\mathrm{C} 84 & \mathrm{H} 91 & \mathrm{~N} 11 & 010 & \mathrm{Cl} 2\end{array}$

$\begin{array}{llll}\mathrm{C} 98 & \mathrm{H} 93 & \mathrm{O} 9 & \mathrm{Cl} 2\end{array}$

$\begin{array}{lllll}\mathrm{C} 97 & \mathrm{H} 87 & \mathrm{~N} 7 & \mathrm{O} 4 & \mathrm{Cl} 2\end{array}$

C91 H81 N13 O8

$\begin{array}{lllll}\mathrm{C} 92 & \mathrm{H} 95 & \mathrm{~N} 8 & \mathrm{O} 2 & \mathrm{Cl}\end{array}$

$\begin{array}{lllll}\mathrm{C} 93 & \mathrm{H} 101 & \mathrm{~N} & \mathrm{O} 7 & \mathrm{Cl}\end{array}$

$\begin{array}{lllll}\mathrm{C} 81 & \mathrm{H} 94 & \mathrm{~N} 12 & 09 & \mathrm{Cl} 3\end{array}$

$\begin{array}{lllll}\mathrm{C} 90 & \mathrm{H} 97 & \mathrm{~N} 5 & \mathrm{O} & \mathrm{Cl}\end{array}$

$\begin{array}{lllll}\mathrm{C} 89 & \mathrm{H} 91 & \mathrm{~N} 12 & \mathrm{O} & \mathrm{Cl} 4\end{array}$

$\mathrm{C} 100 \mathrm{H} 84 \mathrm{~N} 6 \quad 05 \mathrm{Cl}$

$\begin{array}{llll}\mathrm{C} 99 & \mathrm{H} 78 & \mathrm{~N} 13 \mathrm{Cl}\end{array}$

$\begin{array}{llll}\mathrm{C} 99 & \mathrm{H} 85 & \mathrm{~N} 10 & \mathrm{Cl} 2\end{array}$

$\begin{array}{lllll}\mathrm{C} 100 & \mathrm{H} 91 & \mathrm{~N} 3 & 05 & \mathrm{Cl} 2\end{array}$

$\begin{array}{lllll}\mathrm{C} 88 & \mathrm{H} 84 & \mathrm{~N} 14 & 07 & \mathrm{Cl}\end{array}$

Compound $1 \mathrm{H} 2$

(High resolution

Mass analysis)

02-Nov-2005

$10: 52: 44$ $\begin{array}{ll}1490.0 & 1491.0\end{array}$ 


\section{Elemental Composition Report}

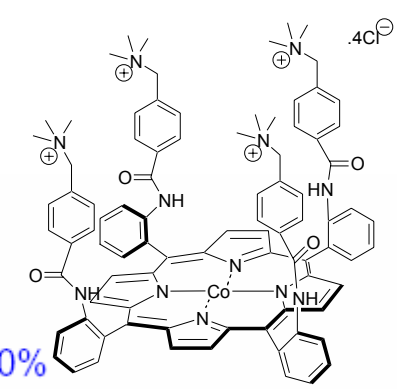

\section{Single Mass Analysis}

Tolerance $=5.0$ PPM / DBE: $\min =-1.5, \max =120.0$

Isotope cluster parameters: Separation $=1.0$ Abundance $=1.0 \%$

Monoisotopic Mass, Odd and Even Electron Ions

8614 formula(e) evaluated with 183 results within limits (up to 140 closest results for each mass)

$$
\begin{aligned}
& \text { Cone } V=30 \\
& \text { Coll } V=10.0
\end{aligned}
$$

Compound $1 \mathrm{Co}$

(High resolution

Mass analysis)

YANY_051102_16244_YAN-4-15V_HRMS 135 (4.578) Cn (Cen,6, 80.00, Ar); Sm (SG, 2x3.00); Sb (2,20.00); Cm (103:142)

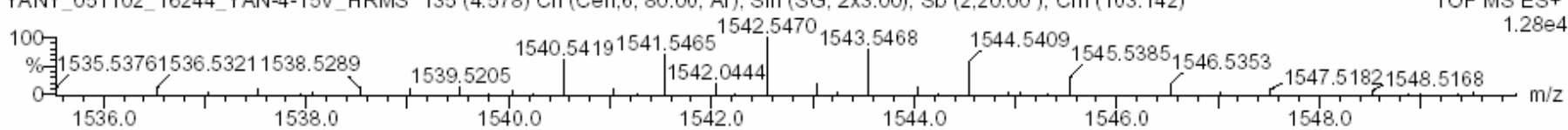

M1nimum :

Maximum:

Mass

1540.5419
Calc. Mass

1540.5419

1540.5417

1540.5417

1540.5421

1540.5422

1540.5422

1540.5422

1540.5422

1540.5415

1540.5423

1540.5424

1540.5414

1540.5414

1540.5414

1540.5413

1540.5426

1540.5426

1540.5427

1540.5427

1540.5410

1540.5410

1540.5428

1540.5428

1540.5409

1540.5409

1540.5409

1540.5408

1540.5408

1540.5431

1540.5431

1540.5432

1540.5405

1540.5404

1540.5404

1540.5404

1540.5434

1540.5435

1540.5436

1540.5436

1540.5402

1540.5437

1540.5400

1540.5400

1540.5399

1540.5399

1540.5439

1540.5441

1540.5441

1540.5441

1540.5397

1540.5397

1540.5396

1540.5442

1540.5442

1540.5395

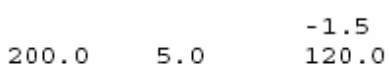

$\mathrm{mDa} \quad \mathrm{PPM}$

DBE

score

Formula

$0.0 \quad 0.0$

0.1

61.5

0.2

0.2

$-0.2$

0.1

49.5

57.5

63.5

53.0

47.5

75.5

45.0

47.5

57.0

51.5

35.5

66.0

41.0

54.0

59.0

53.5

40.5

65.5

52.0

60.0

52.5

47.0

70.5

45.5

48.0

58.5

64.0

54.5

49.0

61.0

56.5

50.0

52.5

58.0

63.0

52.5

50.0

44.5

48.0

56.5

36.0

66.5

54.5

62.5

58.5

70.5

40.0

65.0

52.5

55.0

60.5

52.0

46.5

40.5

64
92
27
135
91
93
127
25
67
3
12
49
112
46
123
121
122
50
108
10
103
71
74
128
29
88
136
137
90
87
56
69
99
23
22
133
96
15
20
61
9
45
115
124
44
119
107
55
106
13
102
101
76
81
35

$\begin{array}{lllll}\mathrm{C} 93 & \mathrm{H} 76 & \mathrm{~N} 13 & \mathrm{O} 6 & \mathrm{Cl} 2\end{array}$

$\begin{array}{llllll}\mathrm{C} 88 & \mathrm{H} 87 & \mathrm{~N} 10 & 08 & \mathrm{Cl} 2 & \mathrm{CO}\end{array}$

$\begin{array}{llllll}\mathrm{C} 99 & \mathrm{H} 88 & \mathrm{~N} 6 & \mathrm{Cl} & \mathrm{CO}\end{array}$

C95 H77 N12 O6 Co

C96 H91 N7 Cl4 Co

$\begin{array}{lllll}\mathrm{C} 97 & \mathrm{H} 97 & \mathrm{O} & \mathrm{Cl} 4 & \mathrm{CO}\end{array}$

C100 H66 N15 O4

$\begin{array}{llllll}\mathrm{C} 85 & \mathrm{H} 90 & \mathrm{~N} 11 & 07 & \mathrm{Cl} 3 & \mathrm{CO}\end{array}$

$\begin{array}{lllll}\mathrm{C} 86 & \mathrm{H} 86 & \mathrm{~N} 11 & 08 & \mathrm{Cl} 4\end{array}$

C90 $\mathrm{H} 79$ N14 O5 $\mathrm{Cl} 3$

$\begin{array}{lllll}\mathrm{C} 91 & \mathrm{H} 85 & \mathrm{~N} 7 & 010 & \mathrm{Cl} 3\end{array}$

$\begin{array}{llllll}\mathrm{C} 81 & \mathrm{H} 97 & \mathrm{~N} 8 & 010 & \mathrm{Cl} 4 & \mathrm{CO}\end{array}$

$\begin{array}{lllll}\mathrm{C} 96 & \mathrm{H} 73 & \mathrm{~N} 12 & 07 & \mathrm{Cl}\end{array}$

$\begin{array}{llllll}\mathrm{C} 80 & \mathrm{H} 91 & \mathrm{~N} 15 & 05 & \mathrm{Cl} 4 & \mathrm{CO}\end{array}$

C91 H84 N9 O9 Cl Co

$\begin{array}{llllll}\mathrm{C} 92 & \mathrm{H} 80 & \mathrm{~N} 13 & \mathrm{O} & \mathrm{Cl} & \mathrm{CO}\end{array}$

$\mathrm{C} 93 \mathrm{H} 86$ N6 $010 \mathrm{Cl}$ Co

$\begin{array}{llllll}\mathrm{C} 82 & \mathrm{H} 93 & \mathrm{~N} 12 & \mathrm{O} 6 & \mathrm{Cl} 4 & \mathrm{CO}\end{array}$

$\begin{array}{lllll}\mathrm{C} 98 & \mathrm{H} 75 & \mathrm{~N} 9 & 08 & \mathrm{Cl}\end{array}$

$\begin{array}{lllll}\mathrm{C} 89 & \mathrm{H} 83 & \mathrm{~N} 10 & 09 & \mathrm{Cl} 3\end{array}$

$\begin{array}{lllll}\mathrm{C} 100 & \mathrm{H} 84 & \mathrm{~N} 6 & \mathrm{O} 2 & \mathrm{Cl} 4\end{array}$

$\begin{array}{lllll}\mathrm{C} 87 & \mathrm{H} 82 & \mathrm{~N} 15 & \mathrm{O} 4 & \mathrm{Cl} 4\end{array}$

$\begin{array}{lllll}\mathrm{C} 88 & \mathrm{H} 88 & \mathrm{~N} 8 & 09 & \mathrm{Cl} 4\end{array}$

C99 H70 N11 O8

$\begin{array}{llllll}\mathrm{C} 83 & \mathrm{H} 88 & \mathrm{~N} 14 & \mathrm{O} 6 & \mathrm{Cl} 3 & \mathrm{CO}\end{array}$

$\begin{array}{llllll}\mathrm{C} 95 & \mathrm{H} 95 & \mathrm{~N} 3 & \mathrm{O} & \mathrm{Cl} & \mathrm{CO}\end{array}$

$\mathrm{C} 94 \mathrm{H} 81$ N8 010 CO

C93 H75 N15 O5 CO

$\begin{array}{llllll}\mathrm{C} 89 & \mathrm{H} 83 & \mathrm{~N} 14 & \mathrm{O} 4 & \mathrm{Cl} 2 & \mathrm{CO}\end{array}$

$\begin{array}{llllll}\mathrm{C} 90 & \mathrm{H} 89 & \mathrm{~N} 7 & \mathrm{O} 9 & \mathrm{Cl} 2 & \mathrm{CO}\end{array}$

$\begin{array}{lllll}\mathrm{C} 95 & \mathrm{H} 78 & \mathrm{~N} 10 & 07 & \mathrm{Cl} 2\end{array}$

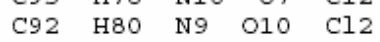

$\begin{array}{llllll}\mathrm{C} 86 & \mathrm{H} 85 & \mathrm{~N} 13 & 07 & \mathrm{Cl} 2 & \mathrm{CO}\end{array}$

$\begin{array}{llllll}\mathrm{C} 98 & \mathrm{H} 92 & \mathrm{~N} 2 & \mathrm{O} & \mathrm{Cl} & \mathrm{CO}\end{array}$

C97 H86 N9 $\mathrm{Cl}_{3} \mathrm{Co}$

$\begin{array}{lllll}\mathrm{C} 97 & \mathrm{H} 79 & \text { N9 } & \text { O7 } & \mathrm{CO}\end{array}$

$\begin{array}{llllll}\mathrm{C} 98 & \mathrm{H} 93 & \mathrm{~N} 4 & \mathrm{O} & \mathrm{Cl} 4 & \mathrm{CO}\end{array}$

$\begin{array}{llllll}\mathrm{C} 86 & \mathrm{H} 86 & \mathrm{~N} 15 & 03 & \mathrm{Cl} 3 & \mathrm{CO}\end{array}$

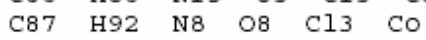

$\begin{array}{lllll}\mathrm{C} 84 & \mathrm{H} 84 & \mathrm{~N} 14 & \mathrm{O} 7 & \mathrm{Cl} 4\end{array}$

$\begin{array}{lllll}\mathrm{C} 92 & \mathrm{H} 81 & \mathrm{~N} 11 & 06 & \mathrm{Cl} 3\end{array}$

$\begin{array}{llllll}\mathrm{C} 79 & \mathrm{H} 95 & \mathrm{~N} 11 & 09 & \mathrm{Cl} 4 & \mathrm{CO}\end{array}$

$\mathrm{C} 94 \mathrm{H} 71$ N15 O6 $\mathrm{Cl}$

$\begin{array}{llllll}\mathrm{C} 89 & \mathrm{H} 82 & \mathrm{~N} 12 & 08 & \mathrm{Cl} & \mathrm{CO}\end{array}$

$\mathrm{C} 100 \quad \mathrm{H} 83$ N8 $\mathrm{O}$ Cl2 $\mathrm{CO}$

$\begin{array}{llllll}\mathrm{C} 94 & \mathrm{H} 82 & \mathrm{~N} 10 & \mathrm{O} & \mathrm{Cl} & \mathrm{CO}\end{array}$

$\begin{array}{lllll}\mathrm{C} 99 & \mathrm{H} 71 & \mathrm{~N} 13 & \mathrm{O} 4 & \mathrm{Cl}\end{array}$

$\begin{array}{llllll}\mathrm{C} 84 & \mathrm{H} 95 & \mathrm{~N} 9 & 07 & \mathrm{Cl} 4 & \mathrm{CO}\end{array}$

$\begin{array}{lllll}\mathrm{C} 100 & \mathrm{H} 77 & \mathrm{~N} 6 & \mathrm{O} & \mathrm{Cl}\end{array}$

$\begin{array}{lllll}\mathrm{C} 87 & \mathrm{H} 81 & \mathrm{~N} 13 & 08 & \mathrm{Cl} 3\end{array}$

$\begin{array}{lllll}\mathrm{C} 99 & \mathrm{H} 88 & \mathrm{~N} 2 & \mathrm{O} & \mathrm{Cl} 4\end{array}$

$\begin{array}{lllll}\mathrm{C} 98 & \mathrm{H} 82 & \mathrm{~N} 9 & \mathrm{C} & \mathrm{Cl} 4\end{array}$

$\begin{array}{lllll}\mathrm{C} 89 & \mathrm{H} 84 & \mathrm{~N} 12 & \mathrm{O} & \mathrm{Cl} 4\end{array}$

$\begin{array}{lllll}\mathrm{C} 90 & \mathrm{H} 90 & \mathrm{~N} 5 & \mathrm{O} 10 & \mathrm{Cl} 4\end{array}$

$\begin{array}{llllll}\mathrm{C} 82 & \mathrm{H} 92 & \mathrm{~N} 10 & 010 & \mathrm{Cl} 3 & \mathrm{CO}\end{array}$ 


\section{Elemental Composition Report}

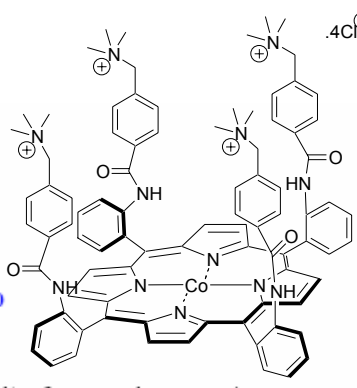

Single Mass Analysis

Tolerance $=5.0$ PPM / DBE: $\min =-1.5, \max =120.0$

Isotope cluster parameters: Separation $=1.0$ Abundance $=1.0 \%$

Monoisotopic Mass, Odd and Even Electron lons

8614 formula(e) evaluated with 183 results within limits (up to 140 closest results for each mass)

Cone $V=30$

Coll $V=10.0$

YANY 051102_16244_YAN-4-15V_HRMS 135 (4.578) Cn (Cen,6, 80.00, Ar); Sm (SG, 2x3.00); Sb (2,20.00); Cm (103:142)

Compound $1 \mathrm{Co}$

(High resolution

Mass analysis)

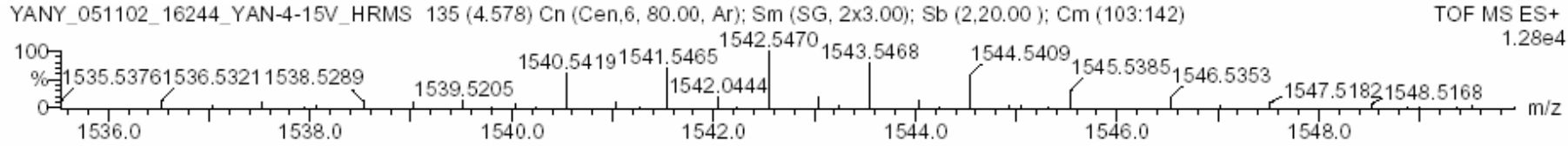

M1nimum :

Maximum :

Mass

Calc. Mass

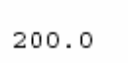

$\begin{array}{ll} & -1.5 \\ 5.0 & 120.0\end{array}$

1540.5395

1540.5395

1540.5394

1540.5444

1540.5444

1540.5445

1540.5445

1540.5392

1540.5390

1540.5448

1540.5448

1540.5449

1540.5449

1540.5449

1540.5450

1540.5450

1540.5387

1540.5387

1540.5386

1540.5386

1540.5385

1540.5453

1540.5453

1540.5454

1540.5454

1540.5383

1540.5383

1540.5455

1540.5382

1540.5382

1540.5382

1540.5381

1540.5458

1540.5458

1540.5459

1540.5459

1540.5378

1540.5378

1540.5378

1540.5377

1540.5377

1540.5377

1540.5461

1540.5462

1540.5463

1540.5375

1540.5464

1540.5464

1540.5373

1540.5372

1540.5372

1540.5466

1540.5466

1540.5466

1540.5467

1540.5467

\begin{tabular}{|c|c|c|c|}
\hline $\mathrm{mDa}$ & PPM & $\mathrm{DBE}$ & score \\
\hline 2.4 & 1.5 & 71.0 & 130 \\
\hline 2.4 & 1.5 & 48.5 & 83 \\
\hline 2.5 & 1.6 & 59.0 & 138 \\
\hline-2.5 & -1.6 & 54.0 & 82 \\
\hline-2.5 & -1.6 & 48.5 & 79 \\
\hline-2.6 & -1.7 & 66.0 & 51 \\
\hline-2.6 & -1.7 & 60.5 & 47 \\
\hline 2.7 & 1.8 & 57.0 & 78 \\
\hline 2.9 & 1.9 & 53.0 & 18 \\
\hline-2.9 & -1.9 & 68.0 & 132 \\
\hline-2.9 & -1.9 & 62.5 & 131 \\
\hline-3.0 & -1.9 & 52.0 & 100 \\
\hline-3.0 & -2.0 & 49.5 & 6 \\
\hline-3.0 & -2.0 & 44.0 & 17 \\
\hline-3.1 & -2.0 & 61.5 & 11 \\
\hline-3.1 & -2.0 & 56.0 & 19 \\
\hline 3.2 & 2.1 & 61.5 & 118 \\
\hline 3.2 & 2.1 & 36.5 & 41 \\
\hline 3.3 & 2.2 & 55.0 & 125 \\
\hline 3.3 & 2.2 & 57.5 & 48 \\
\hline 3.4 & 2.2 & 63.0 & 52 \\
\hline-3.4 & -2.2 & 63.5 & 116 \\
\hline-3.4 & -2.2 & 58.0 & 117 \\
\hline-3.5 & -2.3 & 45.0 & 58 \\
\hline-3.5 & $-2 \cdot 3$ & 39.5 & 60 \\
\hline 3.6 & 2.3 & 55.5 & 98 \\
\hline 3.6 & 2.3 & 61.0 & 97 \\
\hline-3.6 & $-2 \cdot 3$ & 51.5 & 84 \\
\hline 3.7 & 2.4 & 41.0 & 36 \\
\hline 3.7 & 2.4 & 43.5 & 80 \\
\hline 3.7 & 2.4 & 49.0 & 75 \\
\hline 3.8 & 2.5 & 59.5 & 139 \\
\hline-3.9 & -2.5 & 59.0 & 77 \\
\hline-3.9 & -2.5 & 53.5 & 73 \\
\hline-4.0 & -2.6 & 65.5 & 43 \\
\hline-4.0 & -2.6 & 60.0 & 42 \\
\hline 4.1 & 2.6 & 57.5 & 85 \\
\hline 4.1 & 2.6 & 60.0 & 34 \\
\hline 4.1 & 2.7 & 65.5 & 33 \\
\hline 4.2 & 2.7 & 45.5 & 104 \\
\hline 4.2 & 2.7 & 48.0 & 16 \\
\hline 4.2 & 2.7 & 53.5 & 7 \\
\hline-4.2 & -2.7 & 67.5 & 129 \\
\hline-4.3 & -2.8 & 49.0 & 4 \\
\hline-4.4 & -2.8 & 43.5 & 14 \\
\hline 4.4 & 2.9 & 43.5 & 54 \\
\hline-4.5 & -2.9 & 61.0 & 21 \\
\hline-4.5 & -2.9 & 55.5 & 24 \\
\hline 4.6 & 3.0 & 62.0 & 120 \\
\hline 4.7 & 3.0 & 52.5 & 53 \\
\hline 4.7 & 3.0 & 58.0 & 57 \\
\hline-4.7 & -3.1 & 63.0 & 114 \\
\hline-4.7 & -3.1 & 57.5 & 113 \\
\hline-4.7 & -3.1 & 55.0 & 140 \\
\hline-4.8 & -3.1 & 44.5 & 62 \\
\hline-4.8 & -3.1 & 39.0 & 66 \\
\hline
\end{tabular}

Formula

$\begin{array}{llll}\mathrm{C} 97 & \mathrm{H} 68 & \mathrm{~N} 14 & \mathrm{O}\end{array}$

$\begin{array}{llllll}\mathrm{C} 93 & \mathrm{H} 93 & \mathrm{~N} 6 & \mathrm{O} 3 & \mathrm{Cl} 4 & \mathrm{CO}\end{array}$

C92 H79 N11 O9 Co

$\begin{array}{llllll}\mathrm{C} 91 & \mathrm{H} 85 & \mathrm{~N} 11 & 05 & \mathrm{Cl} 2 & \mathrm{CO}\end{array}$

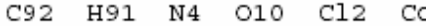

$\begin{array}{lllll}\mathrm{C} 96 & \mathrm{H} 74 & \mathrm{~N} 14 & \mathrm{O} 3 & \mathrm{Cl} 2\end{array}$

$\begin{array}{lllll}\mathrm{C} 97 & \mathrm{H} 80 & \mathrm{~N} 7 & \mathrm{O} & \mathrm{Cl} 2\end{array}$

$\begin{array}{lllll}\mathrm{C} 90 & \mathrm{H} 78 & \mathrm{~N} 12 & 09 & \mathrm{Cl} 2\end{array}$

$\begin{array}{llllll}\mathrm{C} 96 & \mathrm{H} 90 & \mathrm{~N} 5 & \mathrm{O} & \mathrm{Cl} & \mathrm{CO}\end{array}$

C98 H75 $\mathrm{N} 13$ O3 $\mathrm{CO}$

C99 H81 N6 O8 Co

$\begin{array}{llllll}\mathrm{C} 100 & \mathrm{H} 95 & \mathrm{~N} & \mathrm{O} 2 & \mathrm{Cl} 4 & \mathrm{CO}\end{array}$

$\begin{array}{llllll}\mathrm{C} 88 & \mathrm{H} 88 & \mathrm{~N} 12 & \mathrm{O} 4 & \mathrm{Cl} 3 & \mathrm{CO}\end{array}$

C89 H94 N5 O9 $\mathrm{Cl} 3$ Co

$\begin{array}{lllll}\mathrm{C} 93 & \mathrm{H} 77 & \mathrm{~N} 15 & \mathrm{O} 2 & \mathrm{Cl} 3\end{array}$

$\begin{array}{lllll}\mathrm{C} 94 & \mathrm{H} 83 & \mathrm{~N} 8 & \mathrm{O} & \mathrm{Cl} 3\end{array}$

$\begin{array}{lllll}\mathrm{C} 93 & \mathrm{H} 75 & \mathrm{~N} 11 & 010 \mathrm{Cl}\end{array}$

$\begin{array}{llllll}\mathrm{C} 77 & \mathrm{H} 93 & \mathrm{~N} 14 & 08 & \mathrm{Cl} 4 & \mathrm{CO}\end{array}$

$\begin{array}{llllll}\mathrm{C} 87 & \mathrm{H} 80 & \mathrm{~N} 15 & 07 & \mathrm{Cl} & \mathrm{CO}\end{array}$

$\begin{array}{llllll}\mathrm{C} 99 & \mathrm{H} 87 & \mathrm{~N} 4 & \mathrm{O} & \mathrm{Cl} 2 & \mathrm{CO}\end{array}$

$\begin{array}{lllll}\mathrm{C} 98 & \mathrm{H} 81 & \mathrm{~N} 11 & \mathrm{Cl} 2 & \mathrm{CO}\end{array}$

$\begin{array}{llllll}\mathrm{C} 95 & \mathrm{H} 78 & \mathrm{~N} 14 & \mathrm{O} 2 & \mathrm{Cl} & \mathrm{Co}\end{array}$

$\begin{array}{llllll}\mathrm{C} 96 & \mathrm{H} 84 & \mathrm{~N} 7 & \mathrm{O} 7 & \mathrm{Cl} & \mathrm{CO}\end{array}$

$\begin{array}{llllll}\mathrm{C} 85 & \mathrm{H} 91 & \mathrm{~N} 13 & \mathrm{O} 3 & \mathrm{Cl} 4 & \mathrm{CO}\end{array}$

$\begin{array}{llllll}\mathrm{C} 86 & \mathrm{H} 97 & \mathrm{~N} 6 & 08 & \mathrm{Cl} & \mathrm{CO}\end{array}$

$\begin{array}{lllll}\mathrm{C} 97 & \mathrm{H} 86 & \mathrm{~N} 5 & \mathrm{O} & \mathrm{Cl} 4\end{array}$

C96 H80 N12 $\mathrm{Cl} 4$

$\begin{array}{lllll}\mathrm{C} 91 & \mathrm{H} 86 & \mathrm{~N} 9 & \mathrm{O} & \mathrm{C} 14\end{array}$

C80 H90 N13 $09 \quad \mathrm{Cl} 3 \quad \mathrm{CO}$

$\begin{array}{llllll}\mathrm{C} 92 & \mathrm{H} 97 & \mathrm{~N} 2 & \mathrm{O} 7 & \mathrm{Cl} 4 & \mathrm{CO}\end{array}$

$\begin{array}{llllll}\mathrm{C} 91 & \mathrm{H} 91 & \mathrm{~N} 9 & \mathrm{O} & \mathrm{Cl} & \mathrm{CO}\end{array}$

C90 $\mathrm{H77}$ N14 O8 CO

$\begin{array}{lllll}\mathrm{C} 92 & \mathrm{H} 81 & \mathrm{~N} 15 & \mathrm{Cl} & \mathrm{CO}\end{array}$

$\begin{array}{llllll}\mathrm{C} 93 & \mathrm{H} 87 & \mathrm{~N} 8 & \mathrm{O} & \mathrm{Cl} 2 & \mathrm{CO}\end{array}$

$\begin{array}{lllll}\mathrm{C} 98 & \mathrm{H} 76 & \mathrm{~N} 11 & \mathrm{O} 4 & \mathrm{Cl} 2\end{array}$

$\begin{array}{lllll}\mathrm{C} 99 & \mathrm{H} 82 & \mathrm{~N} 4 & \mathrm{O} & \mathrm{Cl} 2\end{array}$

$\begin{array}{lllll}\mathrm{C} 88 & \mathrm{H} 76 & \mathrm{~N} 15 & 08 & \mathrm{Cl} 2\end{array}$

$\begin{array}{lllll}\mathrm{C} 100 & \mathrm{H} 83 & \mathrm{~N} 4 & \mathrm{O} & \mathrm{Cl}\end{array}$

$\begin{array}{llllll}\mathrm{C} 99 & \mathrm{H} 77 & \mathrm{~N} 11 & \mathrm{C} & \mathrm{Cl} 3\end{array}$

$\begin{array}{llllll}\mathrm{C} 83 & \mathrm{H} 87 & \mathrm{~N} 12 & 010 & \mathrm{Cl} 2 & \mathrm{CO}\end{array}$

$\begin{array}{llllll}\mathrm{C} 95 & \mathrm{H} 94 & \mathrm{~N} & 08 & \mathrm{Cl} 3 & \mathrm{CO}\end{array}$

$\begin{array}{llllll}\mathrm{C} 94 & \mathrm{H} 88 & \mathrm{~N} 8 & \mathrm{O} & \mathrm{Cl} 3 & \mathrm{CO}\end{array}$

$\begin{array}{lllll}\mathrm{C} 100 & \mathrm{H} 77 & \mathrm{~N} 10 & \mathrm{CO}\end{array}$

C90 H90 N9 O5 Cl3 Co

$\begin{array}{llllll}\mathrm{C} 91 & \mathrm{H} 96 & \mathrm{~N} 2 & 010 & \mathrm{Cl} 3 & \mathrm{CO}\end{array}$

$\begin{array}{lllll}\mathrm{C} 81 & \mathrm{H} 86 & \mathrm{~N} 13 & 010 & \mathrm{Cl} 4\end{array}$

$\begin{array}{lllll}\mathrm{C} 95 & \mathrm{H} 79 & \mathrm{~N} 12 & \mathrm{O} & \mathrm{Cl} 3\end{array}$

C96 H85 N5 O8 Cl3

$\begin{array}{lllll}\mathrm{C} 91 & \mathrm{H} 73 & \mathrm{~N} 14 & 09 & \mathrm{Cl}\end{array}$

$\begin{array}{lllll}\mathrm{C} 98 & \mathrm{H} 91 & \mathrm{O} 9 & \mathrm{Cl} 2 & \mathrm{CO}\end{array}$

$\begin{array}{llllll}\mathrm{C} 97 & \mathrm{H} 85 & \mathrm{~N} 7 & \mathrm{O} 4 & \mathrm{Cl} 2 & \mathrm{CO}\end{array}$

$\begin{array}{llllll}\mathrm{C} 97 & \mathrm{H} 80 & \mathrm{~N} 11 & \mathrm{O} 3 & \mathrm{Cl} & \mathrm{CO}\end{array}$

$\begin{array}{llllll}\mathrm{C} 98 & \mathrm{H} 86 & \mathrm{~N} 4 & \mathrm{O} 8 \mathrm{Cl} & \mathrm{CO}\end{array}$

C86 H79 N15 O10 Co

$\begin{array}{llllll}\mathrm{C} 87 & \mathrm{H} 93 & \mathrm{~N} 10 & 04 & \mathrm{Cl} 4 & \mathrm{CO}\end{array}$

$\begin{array}{llllll}\mathrm{C} 88 & \mathrm{H} 99 & \mathrm{~N} 3 & 09 & \mathrm{Cl} 4 & \mathrm{CO}\end{array}$ 
Single Mass Analysis

Tolerance $=5.0$ PPM / DBE: $\min =-1.5, \max =120.0$

Isotope cluster parameters: Separation $=1.0$ Abundance $=1.0 \%$

8614 formula(e) evaluated with 183 results within limits (up to 140 closest results for each mass)

Mass analysis)

$$
\begin{aligned}
& \text { Cone } V=30 \\
& \text { Coll } V=10.0
\end{aligned}
$$

YANY_051102_16244_YAN-4-15V_HRMS 135 (4.578) Cn (Cen,6, 80.00, Ar); Sm (SG, 2x3.00); Sb (2,20.00); Cm (103:142)

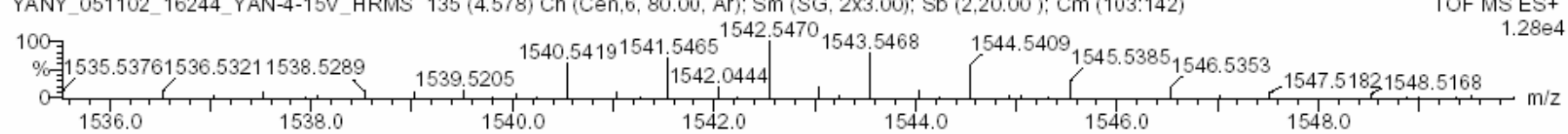

M1nimum :

Maximum :

Mass

\begin{tabular}{|c|c|c|c|c|}
\hline & 200.0 & 5.0 & $\begin{array}{l}-1.5 \\
120.0\end{array}$ & \\
\hline Calc. Mass & $\mathrm{mDa}$ & PPM & DBE & score \\
\hline 1540.5370 & 4.9 & 3.2 & 50.5 & 95 \\
\hline 1540.5370 & 4.9 & 3.2 & 56.0 & 94 \\
\hline 1540.5469 & -5.0 & -3.2 & 56.5 & 86 \\
\hline 1540.5469 & -5.0 & -3.2 & 51.0 & 89 \\
\hline 1540.5369 & 5.0 & 3.3 & 66.5 & 134 \\
\hline 1540.5368 & 5.1 & 3.3 & 44.0 & 72 \\
\hline 1540.5368 & 5.1 & 3.3 & 49.5 & 70 \\
\hline 1540.5367 & 5.2 & 3.4 & 62.5 & 109 \\
\hline 1540.5367 & 5.2 & 3.4 & 68.0 & 110 \\
\hline 1540.5471 & -5.2 & -3.4 & 58.5 & 68 \\
\hline 1540.5471 & -5.2 & -3.4 & 53.0 & 65 \\
\hline 1540.5472 & -5.3 & -3.5 & 70.5 & 40 \\
\hline 1540.5472 & -5.3 & -3.5 & 65.0 & 39 \\
\hline 1540.5472 & -5.3 & -3.5 & 32.0 & 37 \\
\hline 1540.5365 & 5.4 & 3.5 & 55.0 & 32 \\
\hline 1540.5365 & 5.4 & 3.5 & 60.5 & 31 \\
\hline 1540.5365 & 5.4 & 3.5 & 66.0 & 28 \\
\hline 1540.5364 & 5.5 & 3.6 & 46.0 & 105 \\
\hline 1540.5364 & 5.5 & 3.6 & 48.5 & 8 \\
\hline 1540.5364 & 5.5 & 3.6 & 54.0 & 2 \\
\hline 1540.5476 & -5.7 & -3.7 & 54.0 & 1 \\
\hline 1540.5476 & -5.7 & -3.7 & 48.5 & 5 \\
\hline 1540.5477 & -5.8 & -3.8 & 60.5 & 26 \\
\hline 1540.5477 & -5.8 & -3.8 & 55.0 & 30 \\
\hline 1540.5360 & 5.9 & 3.8 & 70.5 & 38 \\
\hline 1540.5359 & 6.0 & 3.9 & 50.5 & 126 \\
\hline 1540.5359 & 6.0 & 3.9 & 53.0 & 59 \\
\hline 1540.5359 & 6.0 & 3.9 & 58.5 & 63 \\
\hline 1540.5480 & -6.1 & -3.9 & 62.5 & 111 \\
\hline
\end{tabular}

Formula

$\begin{array}{lllll}\mathrm{C} 96 & \mathrm{H} 90 & \mathrm{~N} & 09 & \mathrm{Cl} 4\end{array}$

$\begin{array}{lllll}\mathrm{C} 95 & \mathrm{H} 84 & \mathrm{~N} 8 & \mathrm{O} 4 & \mathrm{Cl} 4\end{array}$

$\begin{array}{lllll}\mathrm{C} 92 & \mathrm{H} 82 & \mathrm{~N} 13 & \mathrm{O} 2 & \mathrm{Cl} 4\end{array}$

$\begin{array}{lllll}\mathrm{C} 93 & \mathrm{H} 88 & \mathrm{~N} 6 & 07 & \mathrm{Cl} 4\end{array}$

C94 H70 N13 O10

$\begin{array}{llllll}\mathrm{C} 90 & \mathrm{H} 95 & \mathrm{~N} 5 & \mathrm{O} & \mathrm{Cl} 4 & \mathrm{CO}\end{array}$

$\begin{array}{lllll}\mathrm{C} 89 & \mathrm{H} 89 & \mathrm{~N} 12 & \mathrm{C} & \mathrm{Cl} 4 \\ \mathrm{CO}\end{array}$

$\begin{array}{llllll}\mathrm{C} 100 & \mathrm{H} 82 & \mathrm{~N} 6 & 05 & \mathrm{Cl} & \mathrm{Co}\end{array}$

$\begin{array}{llllll}\mathrm{C} 99 & \mathrm{H} 76 & \mathrm{~N} 13 & \mathrm{Cl} & \mathrm{CO} & \\ \mathrm{C} 94 & \mathrm{H} 83 & \mathrm{~N} 12 & \mathrm{O} 2 & \mathrm{Cl} 2 & \mathrm{CO}\end{array}$

$\begin{array}{llllll}\mathrm{C} 94 & \mathrm{H} 83 & \mathrm{~N} 12 & \mathrm{O} 2 & \mathrm{Cl2} & \mathrm{CO} \\ \mathrm{C} 95 & \mathrm{H} 89 & \mathrm{~N} 5 & \mathrm{O} 7 & \mathrm{Cl2} & \mathrm{CO}\end{array}$

$\begin{array}{llll}\mathrm{C} 99 & \mathrm{H} 72 & \mathrm{~N} 15 & \mathrm{Cl} 2\end{array}$

$\begin{array}{lllll}\mathrm{C} 100 & \mathrm{H} 78 & \mathrm{~N} 8 & \mathrm{O} & \mathrm{Cl} 2\end{array}$

$\begin{array}{llllll}\mathrm{C} 73 & \mathrm{H} 95 & \mathrm{~N} 15 & 010 & \mathrm{Cl} 4 & \mathrm{CO}\end{array}$

$\begin{array}{llll}\mathrm{C} 99 & \mathrm{H} 87 & 010 & \mathrm{Cl} 3\end{array}$

$\begin{array}{lllll}\mathrm{C} 98 & \mathrm{H} 81 & \mathrm{~N} 7 & \mathrm{O} & \mathrm{Cl}\end{array}$

$\begin{array}{llll}\mathrm{C} 97 & \mathrm{H} 75 & \mathrm{~N} 14 & \mathrm{Cl} 3\end{array}$

$\begin{array}{llllll}\mathrm{C} 81 & \mathrm{H} 85 & \mathrm{~N} 15 & 09 & \mathrm{Cl} 2 & \mathrm{CO}\end{array}$

$\begin{array}{lllllll}\mathrm{C} 93 & \mathrm{H} 92 & \mathrm{~N} 4 & \mathrm{O} 7 & \mathrm{Cl} 3 & \mathrm{CO}\end{array}$

$\begin{array}{llllll}\mathrm{C} 92 & \mathrm{H} 86 & \mathrm{~N} 11 & \mathrm{O} 2 & \mathrm{Cl} 3 & \mathrm{CO}\end{array}$

$\begin{array}{llllll}\mathrm{C} 91 & \mathrm{H} 86 & \mathrm{~N} 13 & \mathrm{C} & \mathrm{C} 3 & \mathrm{CO}\end{array}$

$\begin{array}{llllll}\mathrm{C} 92 & \mathrm{H} 92 & \mathrm{~N} 6 & \mathrm{C} & \mathrm{C} 3 & \mathrm{CO}\end{array}$

$\begin{array}{lllll}\mathrm{C} 97 & \mathrm{H} 81 & \mathrm{~N} 9 & \mathrm{O} 4 & \mathrm{Cl} 3\end{array}$

$\begin{array}{lllll}\mathrm{C} 98 & \mathrm{H} 87 & \mathrm{~N} 2 & \mathrm{O} 9 & \mathrm{Cl}\end{array}$

$\mathrm{C} 100 \mathrm{H} 72 \mathrm{~N} 13 \circ \mathrm{Cl} 2$

$\begin{array}{llllll}\mathrm{C} 84 & \mathrm{H} 82 & \mathrm{~N} 14 & 010 & \mathrm{Cl} & \mathrm{CO}\end{array}$

$\begin{array}{llllll}\mathrm{C} 96 & \mathrm{H} 89 & \mathrm{~N} 3 & \mathrm{O} & \mathrm{Cl} 2 & \mathrm{CO}\end{array}$

$\begin{array}{llllll}\mathrm{C} 95 & \mathrm{H} 83 & \mathrm{~N} 10 & \mathrm{O} & \mathrm{Cl} 2 & \mathrm{CO}\end{array}$

C99 H82 N8 O4 Cl Co 
X-band (9.37GHz) EPR spectra of 1 Co buffer solutions $(\mathrm{C}>1 \mathrm{mM})$ under different conditions, recorded at $4 \mathrm{~mW}$ and $77 \mathrm{~K}$ :

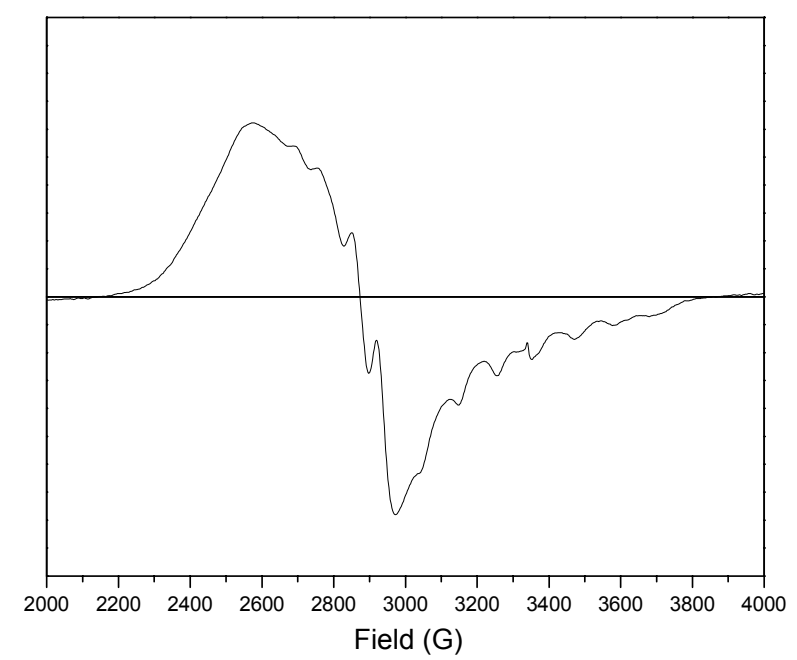

Figure $\mathrm{S} 1$. In the absence of ligand 2, at $\mathrm{pH} 7.0$, under $\mathrm{N}_{2}, \mathrm{~g}_{\|}=2.02, \mathrm{~A}_{\|}=110 \mathrm{G}$, $\mathrm{g}_{\perp}$ is not resovled.
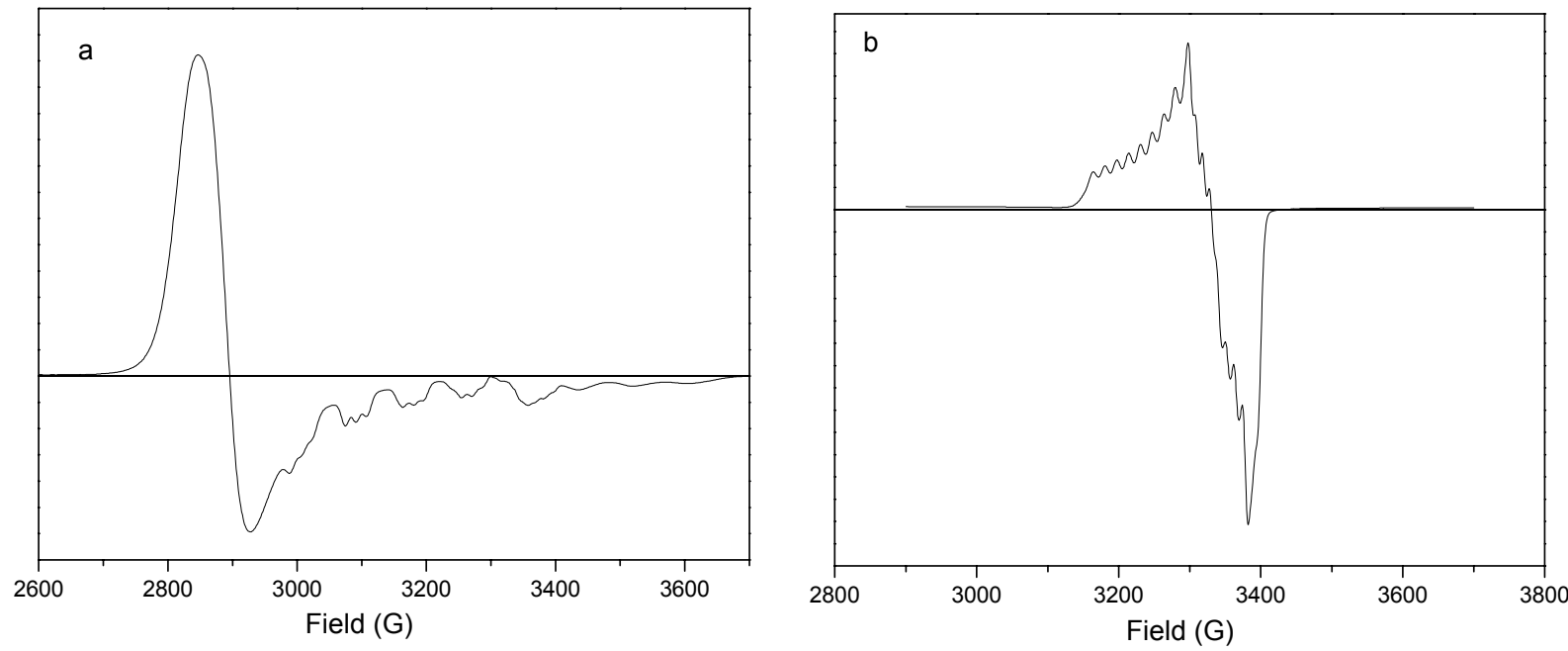

Figure S2. (a)In the presence of ligand 2, at $\mathrm{pH} 7.0$, under $\mathrm{N}_{2}, \mathrm{~g}_{\|}=2.02, \mathrm{~A}_{\|}=86 \mathrm{G}, \mathrm{A}_{\mathrm{N}}$ $=16 \mathrm{G}, \mathrm{g}_{\perp}=2.31$; (b) In the presence of ligand 2, at $\mathrm{pH} 7.0$, under $\mathrm{O}_{2}, \mathrm{~g}_{\|}=2.08, \mathrm{~A}_{\|}=$ $17 \mathrm{G}, \mathrm{g}_{\perp}=2.01, \mathrm{~A}_{\perp}=11 \mathrm{G}$. Spin quantitation shows a:b 100:66 (intensity lost over time). 

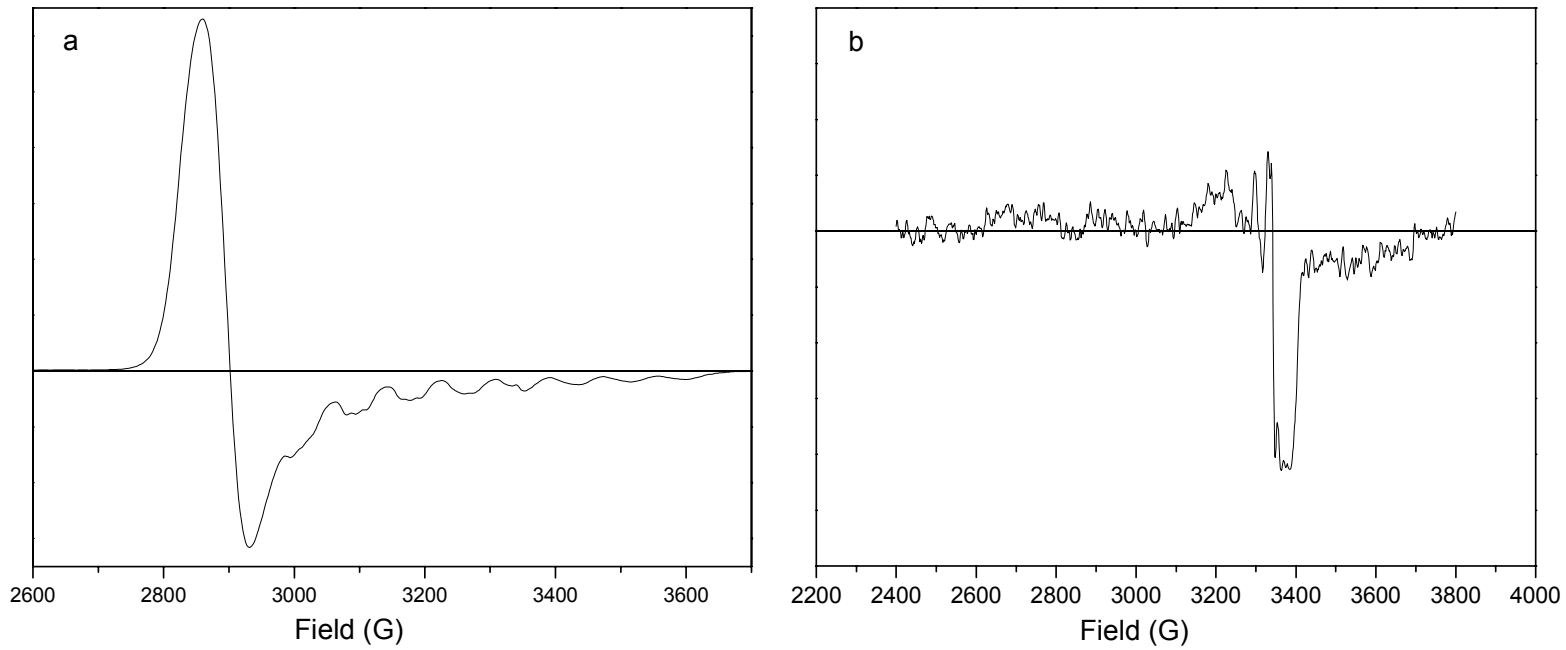

Figure S3. (a) In the presence of ligand 2, at $\mathrm{pH} 5.0$, under $\mathrm{N}_{2}, \mathrm{~g}_{\|}=2.02, \mathrm{~A}_{\|}=85 \mathrm{G}$, $\mathrm{A}_{\mathrm{N}}=15 \mathrm{G}, \mathrm{g}_{\perp}=2.31$; (b) In the presence of ligand 2 , at $\mathrm{pH} 5.0$, under $\mathrm{O}_{2}$ (oxygenation adduct decomposed). Spin quantitation shows a:b 100:0.7.
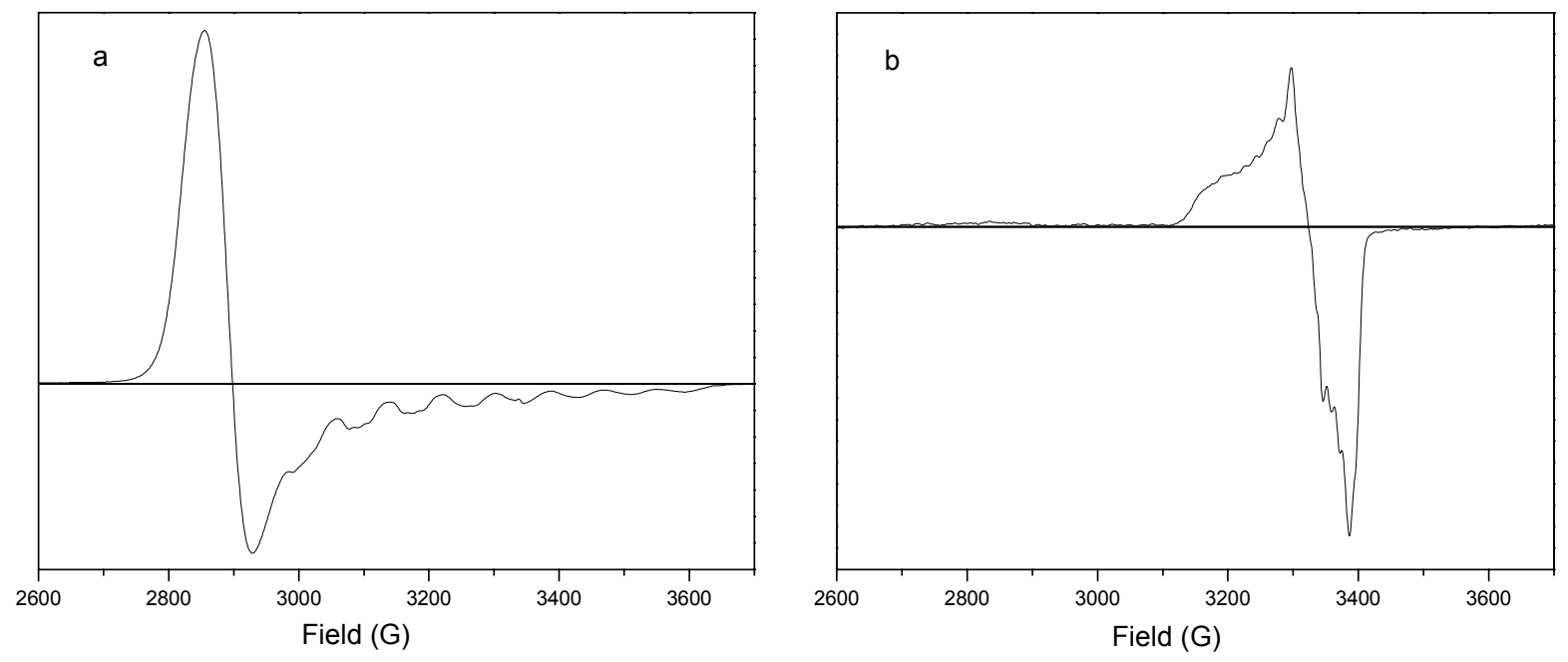

Figure S4. (a) In the presence of ligand 2, at $\mathrm{pH} 9.0$, under $\mathrm{N}_{2}, \mathrm{~g}_{\|}=2.02, \mathrm{~A}_{\|}=83 \mathrm{G}$, $\mathrm{A}_{\mathrm{N}}=15 \mathrm{G}, \mathrm{g}_{\perp}=2.31$; (b) In the presence of ligand 2 , at $\mathrm{pH} 9.0$, under $\mathrm{O}_{2}, \mathrm{~g}_{\|}=2.08, \mathrm{~A}_{\|}$ $=17 \mathrm{G}, \mathrm{g}_{\perp}=2.01, \mathrm{~A}_{\perp}=11 \mathrm{G}$. Spin quantitation shows $\mathrm{a}: \mathrm{b} \sim 100: 4$. 